\title{
A dynamic optimal control model for COVID-19 and cholera co-infection in Yemen
}

Ibrahim M. Hezam ${ }^{1,2^{*}}$ (D), Abdelaziz Foul ${ }^{1}$ and Adel Alrasheedi ${ }^{1}$

\section{"Correspondence:}

ialmishnanah@ksu.edu.sa

1 Statistics and Operations Research Department, College of Sciences,

King Saud University, Riyadh, Saudi Arabia

${ }^{2}$ Department of Mathematics, Ibb

University, Ibb, Yemen

\section{Springer}

\begin{abstract}
In this work, we propose a new dynamic mathematical model framework governed by a system of differential equations that integrates both COVID-19 and cholera outbreaks. The estimations of the model parameters are based on the outbreaks of COVID-19 and cholera in Yemen from January 1, 2020 to May 30, 2020. Moreover, we present an optimal control model for minimizing both the number of infected people and the cost associated with each control. Four preventive measures are to be taken to control the outbreaks: social distancing, lockdown, the number of tests, and the number of chlorine water tablets (CWTs). Under the current conditions and resources available in Yemen, various policies are simulated to evaluate the optimal policy. The results obtained confirm that the policy of providing resources for the distribution of CWTs, providing sufficient resources for testing with an average social distancing, and quarantining of infected individuals has significant effects on flattening the epidemic curves.
\end{abstract}

Keywords: Cholera; Co-infection; COVID-19; Optimal control; Yemen

\section{Introduction}

Recently, Yemen has suffered from many disasters and fierce armed conflicts. It is reported by the UN that Yemen is going through one of the worst humanitarian crises all over the world. The political armed conflict has led to a humanitarian crisis in all fields of life, i.e., more than $85 \%$ of the population are suffering from the lack of basic essentials such as food, water, electricity, and medicines. The scarcity of drinkable water has resulted in the spread of infectious diseases such as cholera.

In Yemen, cholera has become the largest epidemic in the modern-day world. According to the World Health Organization (WHO), the cumulative total number of suspected infected individuals from January 2018 to May 2020 is $(1,371,819)$ with 1566 deaths [1]. Cholera is a water-borne bacterial disease which can be transmitted to humans either through humans or water. Several works have investigated the epidemic model of cholera infections, such as Tian and Wang in [2], which implemented some epidemic models for cholera through mathematical analysis. Moreover, the endemic global stability was investigated using three techniques: monotonic dynamical systems, geometric approach, and Lyapunov functions, up to July 17, 2020. Another study is that by Dangbé et al. [3] which identified climatic factors and human behavior parameters that minimize the spread of

(c) The Author(s) 2021. This article is licensed under a Creative Commons Attribution 4.0 International License, which permits use sharing, adaptation, distribution and reproduction in any medium or format, as long as you give appropriate credit to the original author(s) and the source, provide a link to the Creative Commons licence, and indicate if changes were made. The images or other third party material in this article are included in the article's Creative Commons licence, unless indicated otherwise in a credit line to the material. If material is not included in the article's Creative Commons licence and your intended use is not permitted by statutory regulation or exceeds the permitted use, you will need to obtain permission directly from the copyright holder. To view a copy of this licence, visit http://creativecommons.org/licenses/by/4.0/. 
cholera tangibly or intangibly. The equilibria stabilities of ordinary differential equations in the proposed model were investigated. This work was also applied to some localities of Cameroon and Chad. Likewise, Kobe et al. in [4] suggested a game model of cholera that allows individuals to select one of two programs: vaccinations or clean water consumption. Another related study is that of NKDO Opoku, and Afriyie, C. in [5]. They developed a mathematical model of cholera transmission dynamics of cholera and investigated the following two control measures: education campaign and treatment of water bodies. Berhe in [6] provided a theoretical study of the optimally controlled model of cholera dynamics. The model's parameters are estimated using cholera data taken from the Oromia region, Ethiopia. Sensitivity analyses were then given for the rate of the infected humans and that of the recovery, as they are the most important parameters. In addition, several techniques were proposed to reduce the number of infected persons and the overall cost associated with each control either separately or both by monitoring the treatment and sanitation parameters.

Furthermore, some studies have discussed the cholera epidemic in Yemen. Based on 2017 real data, Nishiura et al. in [7] predicted the peak of cholera epidemic in Yemen. To estimate the final epidemic size, they used logistic and generalized logistic models. In the same manner, Yang and Wang in [8] updated the susceptible-infected-recovered (SIR) model to include a parameter measuring the availability of medical resources and facilities. They also provided a mathematical analysis of the proposed model. The proposed model was implemented on the real data taken from a cholera epidemic in Yemen from April 2017 to May 2018. Enhancing the same study, Lemos-Paião et al. in [9] introduced vaccination to the SIR model as a parameter for optimal control. This model was applied to the cholera outbreak in Yemen from April 27, 2017 to April 15, 2018. Based on the same data taken in the same period, Lemos-Paião et al. in [10] updated the SIR model to require quarantining during the treatment period. Also, one parameter measure was used for optimal control, corresponding to the ratio of susceptible individuals receiving the chlorine water tablets (CWTs) for water purification. The next related study concering cholera outbreak in Yemen in the same period was carried by Carfora and Torcicollo in [11]. In their study, the SIR model considered parameters that reflect direct (human-to-human) and indirect (environment-to-human) spread. The approximation approach of the least squares was also used to estimate the epidemiological parameters.

All the above studies have to do with cholera, on the other side; there are some that appeared with the outbreak of COVID-19. The first confirmed case of COVID-19 in Yemen was discovered in April 2020. Since then, the cumulative total number of infected cases and deaths due to COVID-19 up to June 17, 2020 are 902 and 244 cases, respectively. It should be noted that the announced cases are much less than the actual number of cases in Yemen due to many reasons (e.g., political or technical). Dozens of mathematical models have been proposed to control the dynamics of the COVID-19 pandemic. Madubueze in [12] updated the SEQIHR (susceptible-exposed-quarantinedinfected-hospitalized-recovered) model to include new parameters reflecting the impact of health education, quarantine, and isolation. These parameters were used to determine the optimal control and to find the minimum cost associated with each control. As to Khajji et al. in [13], the proposed mathematical model included the dynamic transmission of COVID-19 between humans and animals in a region or in several regions at discrete times. They presented some control strategies to protect the maximum number of individuals. 
Hence, the least cost and most effective strategy to be determined. Perkins and Espana in [14] presented an optimal control analysis of the susceptible-exposed-asymptomaticinfected-hospitalized-vaccinated (SEAIHV) model of COVID-19 dynamic transmission. They also added a parameter representing non-pharmaceutical interventions. The optimal control priority was to minimize the total number of deaths versus reduced time under control. The validity of the model was tested based on the real data taken from US, from May 2020 through December 2021. Likewise, Wickramaarachchi and Perera in 2020 [15] expanded the susceptible-exposed-infected-recovered (SEIR) model. They classified the infected people into four groups: asymptomatic patients, patients with mild symptoms, hospitalized patients, and critical patients. They also used two parameters to reflect personal protection rate: asymptomatic people identification rate and tracking rate. The proposed model was implemented to fight the COVID-19 outbreak in Sri Lanka. Yousefpour et al. [16] proposed the susceptible-exposed-asymptomatic-infectedhospitalized-recovered (SEAIHR) model. They designed a multi-objective genetic algorithm for optimal control of economic consequence strategies. Tsay et al. in [17] expanded the susceptible-exposed-asymptomatic-infected-removed (SEAIHR) model to include the perished class due to COVID-19 infection. Besides, an analytical comparison was presented to estimate the system parameters for USA, Italy, Spain, and Germany. Social distancing, extensive testing, and quarantining were used for dynamic optimal control. The model aimed at minimizing social and economic costs so as to maintain the size of the epidemic below its specific peak value. Also, the authors in [18-21] presented interesting mathematical models that discussed controlling the spread of COVID-19 and allocation of COVID-19 vaccines to priority groups using the MCDM approach. Besides, several studies have provided mathematical analyses of other infectious diseases such as TB [22-24], Ebola [25], HBV [26], HIV [27], Lassa hemorrhagic fever [28], dengue [29], etc.

Recently, some studies on co-infection models have been carried out. Co-infection is simultaneous infection of an individual due to the outbreak of more than one infectious disease in the same place. The first related study to be indicated here is that of $\mathrm{Li}$ et al. [30]. They proposed an epidemiological model concerned with co-infection with two diseases where one of them is chronic, the other is acute. Similarly, Gao et al. [31] developed and analyzed a simple susceptible-infected-susceptible (SIS) model of co-infections. Similarly, Tang et al. [32] proposed a mathematical model of dual-infection of dengue and Zika virus. Again, Ghersheen et al. [33, 34] proposed SIR models to describe dual infection in the same area. Also, Khan et al. [35] proposed a co-infection model that includes the Atangana-Baleanu fractional derivative, which is a combination of the human immunodeficiency virus (HIV) and tuberculosis (TB). Besides, Mushayabasa and Bhunu [36] proposed a mathematical model concerning the relationship between HIV and cholera outbreaks. Further, Okosun and Makinde in [37] formulated an SIR model for the concurrent infection of malaria and cholera. The authors discussed five parameters for optimal control: two of them were for preventing both diseases, the other two were for controlling the treatment of each disease, and the last one for controlling the co-infection treatment. Equally important, Okosun et al. [38] proposed a compartmental model to address the co-infection dynamics of cholera and schistosomiasis diseases. In the same way, Marimuthu et al. [39] investigated the impact of COVID-19 on TB patients using the SEIR model in India. According to public health interventions, two policies were 
studied to estimate the basic reproduction number. Lam et al. [40] wrote a letter for controlling the simultaneous outbreak of dengue and COVID-19 in Singapore. Doungmo et al. in [41] discussed the coinfection of HIV with the COVID-19 based on a mathematical model, and Hezam in [42] combined the COVID-19 model and the unemployment problem, while Zhang and Jain in [43] investigated the transmission of the Ebola and the Covid-19 viruses.

However, studies on mathematical models of cholera infection with other infectious diseases are rare. At the time of writing this paper, mathematical model studies of COVID-19 infection with other diseases are almost scarce. Besides, there is no epidemiological model in the literature so far that implements the co-infection dynamics of cholera and COVID19. On the other hand, the simultaneous outbreak of both diseases in Yemen overwhelms the fragile health care system. In fact, the control variables used to encounter any infection contribute indirectly to fighting another infection. Filling swamps, purifying water along with pure environment and personal hygiene, for example, assist to curb cholera outbreak, on one hand, and on the other they are significant factors in fighting COVID-19. Likewise, social distancing leads to curbing COVID-19 and helps also to control cholera outbreak. Therefore, we need to propose an optimal control model that combines two simultaneous epidemics in the same region. Hence, this is the founding motivation of this study.

In this study, we present a formulation consisting of dynamic ordinary differential equations of an epidemiological co-infection model. This work provides the following significant contributions. Firstly, we propose a framework for mathematical model that integrates COVID-19 and cholera diseases. Secondly, based on the actual data about both infections from January 1, 2020 to May 30, 2020, we estimate parameters so as to predict the trajectories of both outbreaks for 100 weeks. Thirdly, we propose an optimal control model to minimize both the expected cumulative number of people infected with COVID19 and cholera, and the total cost associated with each control. Fourthly, by testing a novel set of policies, we examine the responsiveness of the optimum of control inputs. We give special emphasis on inputs related to social distancing, lockdown, number of tests, and chlorine availability in order to determine the optimal policies that lead to infection mitigation. Fifthly, all the policies that have to do with COVID-19 and cholera outbreaks in Yemen are carried out. Ultimately, under the current conditions and available resources in Yemen, we determine the optimal policy.

What follows in this work is organized as follows. Section 2 discusses the co-infection model formulation. Section 3 discusses the estimated parameters problem. In Sect. 4, the optimal control model and its analysis are provided. In Sect. 5, numerical simulations are presented. Finally, in Sect. 6, the work is summarized and concluded.

\section{Model formulation}

This study builds upon the models presented in [10, 11, 17]. Tsay et al. in [17] discussed dynamic models of COVID-19, and other studies addressed cholera outbreak in Yemen. To investigate the co-infection dynamics of COVID-19 and cholera, we subdivide the total human population into fourteen different epidemiological classes whose descriptions are found in Table 1. Furthermore, Table 2 summarizes the parameters used in the coinfection model. 
Table 1 Descriptions and initial values of model variables

\begin{tabular}{llll}
\hline Variables & Description & Initial conditions & Source \\
\hline$N$ & Total population size & $29,825,964$ & {$[44]$} \\
$S$ & Susceptible to both COVID-19 and cholera & $N$ & Assumed \\
$E$ & Number of exposed to COVID-19 & {$\left[0, N \times 10^{-6}\right]$} & {$[17]$} \\
$A$ & Number of asymptomatic COVID-19 individuals & 0 & Assumed \\
$I_{1}$ & Number of COVID-19- infected individuals & 0 & Assumed \\
$R_{1}$ & Recovered from COVID-19 & 0 & Assumed \\
$P_{1}$ & Perished by COVID-19 & 0 & Assumed \\
$I_{R 2}$ & Infected with cholera after recovery from COVID-19 & 0 & Assumed \\
$I_{2}$ & Infected with cholera & $I_{0}=750$ person & {$[10]$} \\
$R_{2}$ & Recovered from cholera & 0 & Assumed \\
$P_{2}$ & Perished by cholera & 0 & Assumed \\
$I_{1 R}$ & Infected with COVID-19 after recovery from cholera & 0 & Assumed \\
$I_{12}$ & Co-infected with both cholera and COVID-19 & 0 & Assumed \\
$R_{12}$ & Recovered from both cholera and COVID-19 & 0 & Assumed \\
$P_{12}$ & Perished by both COVID-19 and cholera & 0 & Assumed \\
$B$ & Bacterial concentration in the environment (free & $275 \times 10^{3}$ (cell/ml) & {$[10]$} \\
& bacteria population living in the environment) & & \\
\hline & & &
\end{tabular}

Table 2 Descriptions and values of model parameters

\begin{tabular}{|c|c|c|c|}
\hline Parameters & Description & Value (range) & Source \\
\hline peak & Peak limit of COVID-19 & {$\left[10^{4}, 10^{5}\right]$} & Assumed \\
\hline$P_{2}^{\text {peak }}$ & Peak limit of cholera & {$\left[10^{5}, 5 \times 10^{5}\right]$} & Assumed \\
\hline$u_{1}(t)$ & $\begin{array}{l}\text { Time-dynamic function to measure the social distancing } \\
\text { rate }\end{array}$ & {$[0.05,0.5]$} & {$[17]$} \\
\hline$u_{2}(t)$ & $\begin{array}{l}\text { Time-dynamic function to measure the quarantining } \\
\text { rate }\end{array}$ & {$[0.01,0.3]$} & [17] \\
\hline$u_{3}(t)$ & Time-dynamic function to measure the testing rate & {$[0.1,0.3]$} & [17] \\
\hline$u_{4}(t)$ & $\begin{array}{l}\text { Time-dynamic function to measure the fraction of } \\
\text { susceptible individuals who have access to CWT for } \\
\text { water purification }\end{array}$ & {$[0.2,1]$} & {$[10]$} \\
\hline$t_{\text {latent }}^{-1}$ & Latent period of the virus & 0.5 days $^{-1}$ & [17] \\
\hline$\alpha_{c}(t)$ & Direct transmission rate of cholera & $5.5 e^{-7}$ & {$[11]$} \\
\hline$\beta$ & Indirect transmission rate of cholera & $0.02325 /$ week & Estimated \\
\hline$K_{B}$ & Half saturation constant & $10^{6}(\mathrm{cell} / \mathrm{ml})$ & [11] \\
\hline$\mu_{1}$ & Susceptible to COVID-19 after recovering & 0 & [17] \\
\hline$\mu_{2}$ & Susceptible to cholera after recovering & 0.0003/week & [11] \\
\hline$\mu_{12}$ & $\begin{array}{l}\text { Susceptible to both COVID-19 and cholera after } \\
\text { recovering }\end{array}$ & 0 & Assumed \\
\hline$\rho$ & $\begin{array}{l}\text { Infectious period for subjects with unconfirmed } \\
\text { infections }\end{array}$ & 0.1 days $^{-1}$ & [17] \\
\hline$\delta_{1}$ & Infection rate with cholera from COVID-19 patient & 0 & Estimated \\
\hline$\beta_{1}, \beta_{2}$ & Recovery rate from COVID-19 and cholera & $0.005,1.5$ & Estimated \\
\hline$\lambda_{1}$ & Infected rate of cholera after recovery from COVID-19 & 0 & Estimated \\
\hline$v_{1}$ & Recovery rate from COVID-19 and cholera sequentially & 0 & Estimated \\
\hline$\omega_{1}, \omega_{2}, \omega_{12}$ & Death rate by COVID-19, cholera, and both & $0.0447,0.0002,0$ & Estimated \\
\hline$\alpha_{12}$ & $\begin{array}{l}\text { Infected rate by both cholera and COVID-19 } \\
\text { simultaneously }\end{array}$ & 0 & Estimated \\
\hline$\delta_{2}$ & Infected rate with COVID-19 from cholera patient. & 0 & Estimated \\
\hline$\xi$ & Shedding rate (infected) & $\begin{array}{l}70\left(\text { cell/ml } \text { week }^{-1}\right. \\
\left.\text { person }^{-1}\right)\end{array}$ & [11] \\
\hline$\tau$ & Bacteria death rate & $0.233 /$ week & [11] \\
\hline$\lambda_{2}$ & Infected rate by COVID-19 after recovering from cholera & 0 & Estimated \\
\hline$v_{2}$ & Recovery rate from cholera and COVID-19 sequentially & 0 & Estimated \\
\hline$v_{12}$ & $\begin{array}{l}\text { Recovery rate from both COVID-19 and cholera } \\
\text { simultaneously }\end{array}$ & 0 & Estimated \\
\hline k & Bacteria carrying capacity & $K_{B} / 2$ & [11] \\
\hline$b$ & Allee threshold when $\tau=0$ & $K_{B} / 10$ & [11] \\
\hline$r$ & Bacterial intrinsic growth rate & 1 & [11] \\
\hline$\Lambda$ & A constant rate of the total population & 0.3 & Assumed \\
\hline
\end{tabular}




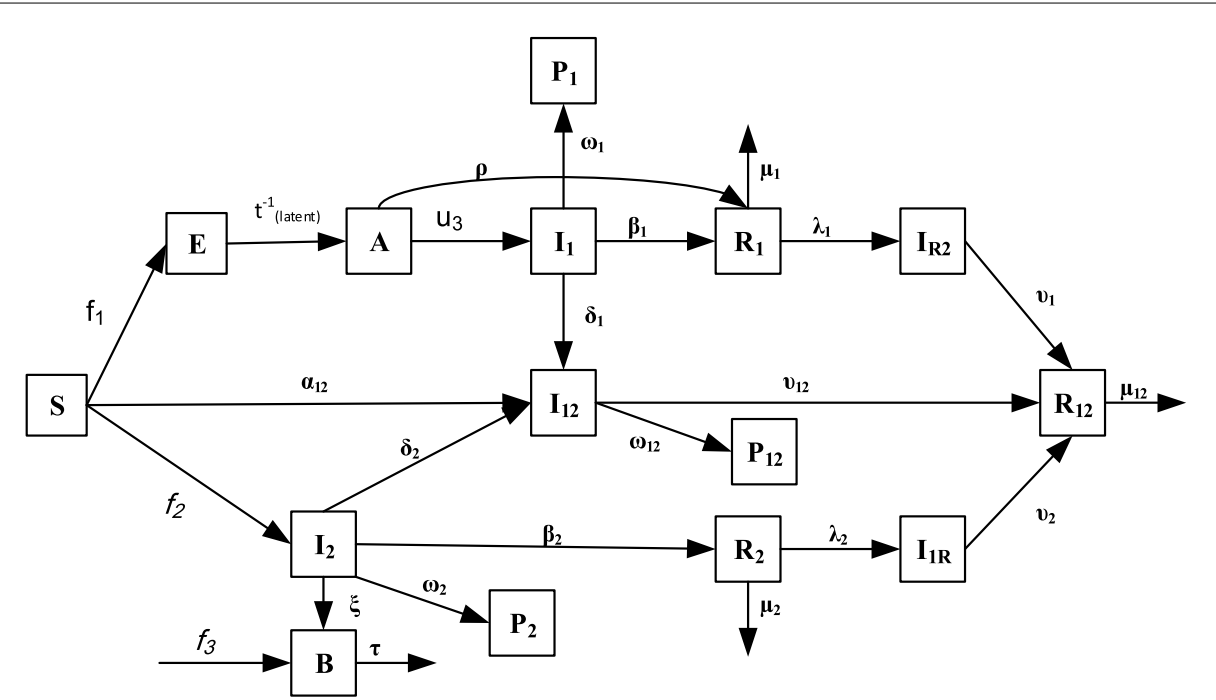

Figure 1 Schematic diagram of the compartmental cholera-COVID-19 co-infection model. Please note that $f_{1}=\left(1-u_{1}(t)\right) A(t)+\left(1-u_{2}(t)\right) I_{1}(t), f_{2}=\alpha_{C}(t) l_{2}(t)+\frac{\beta B}{K_{B}+B}\left(1-u_{4}(t)\right)$ and $f_{3}=r B(B-b)\left(1-\frac{B}{k}\right)$ in the flow chart

The variables $S(t), E(t), A(t), I_{1}(t), R_{1}(t), P_{1}(t), I_{R 2}(t), I_{2}(t), R_{2}(t), P_{2}(t), I_{1 R}(t), I_{12}(t)$, $R_{12}(t)$, and $P_{12}(t)$ correspond to the numbers of individuals in the fourteen epidemiological classes at time $t$. The total human population at time $t$, denoted by $X(t)$, is given by

$$
\begin{aligned}
X(t)= & S(t)+E(t)+A(t)+I_{1}(t)+R_{1}(t)+P_{1}(t)+I_{R 2}(t)+I_{2}(t)+R_{2}(t)+P_{2}(t) \\
& +I_{1 R}(t)+I_{12}(t)+R_{12}(t)+P_{12}(t) .
\end{aligned}
$$

Moreover, the total human population is combined with the pathogen population $B(t)$ in the environment, where $B(t)$ reflects the bacterial concentration for cholera infection dynamics at time $t$.

The schematic diagram of the compartmental COVID-19-cholera co-infection model is shown in Fig. 1.

The proposed co-infection model is described by the following system of equations:

$$
\begin{aligned}
\frac{d S(t)}{d t}= & -\left(\frac{\left(1-u_{1}(t)\right)}{N} A(t)+\frac{\left(1-u_{2}(t)\right)}{N} I_{1}(t)+\alpha_{c}(t) I_{2}(t)\right. \\
& \left.+\frac{\beta B}{K_{B}+B}\left(1-u_{4}(t)\right)+\alpha_{12}\right) S(t)+\mu_{1} R_{1}(t)+\mu_{2} R_{2}(t)+\mu_{12} R_{12}(t)+\Lambda, \\
\frac{d E(t)}{d t}= & \left(\frac{\left(1-u_{1}(t)\right)}{N} A(t)+\frac{\left(1-u_{2}(t)\right)}{N} I_{1}(t)\right) S(t)-t_{\text {latent }}^{-1} E(t), \\
\frac{d A(t)}{d t}= & t_{\text {latent }}^{-1} E(t)-\left(u_{3}(t)+\rho\right) A(t), \\
\frac{d I_{1}(t)}{d t}= & u_{3}(t) A(t)-\left(\delta_{1}+\beta_{1}+\omega_{1}\right) I_{1}(t), \\
\frac{d R_{1}(t)}{d t}= & \rho A(t)+\beta_{1} I_{1}(t)-\left(\mu_{1}+\lambda_{1}\right) R_{1}(t),
\end{aligned}
$$




$$
\begin{aligned}
& \frac{d P_{1}(t)}{d t}=\omega_{1} I_{1}(t) \\
& \frac{d I_{R 2}(t)}{d t}=\lambda_{1} R_{1}(t)-v_{1} I_{R 2}(t), \\
& \frac{d I_{12}(t)}{d t}=\alpha_{12} S(t)+\delta_{1} I_{1}(t)+\delta_{2} I_{2}(t)-\left(v_{12}+\omega_{12}\right) I_{12}(t), \\
& \frac{d R_{12}(t)}{d t}=v_{1} I_{R 2}(t)+v_{2} I_{1 R}(t)+v_{12} I_{12}(t)-\mu_{12} R_{12}(t), \\
& \frac{d P_{12}(t)}{d t}=\omega_{12} I_{12}(t), \\
& \frac{d I_{2}(t)}{d t}=\left(\alpha_{c}(t) I_{2}(t)+\frac{\beta B}{K_{B}+B}\left(1-u_{4}(t)\right)\right) S(t)-\left(\delta_{2}+\beta_{2}+\omega_{2}+\xi\right) I_{2}(t), \\
& \frac{d B(t)}{d t}=r B(B-b)\left(1-\frac{B}{k}\right)-\tau B+\xi I_{2}(t), \\
& \frac{d R_{2}(t)}{d t}=\beta_{2} I_{2}(t)-\left(\mu_{2}+\lambda_{2}\right) R_{2}(t), \\
& \frac{d P_{2}(t)}{d t}=\omega_{2} I_{2}(t), \\
& \frac{d I_{1 R}(t)}{d t}=\lambda_{2} R_{2}(t)-v_{2} I_{1 R}(t) .
\end{aligned}
$$

Equation (1) describes individuals susceptible to both COVID-19 and cholera. Inputs for this epidemiological class are the fraction of the total population at a constant rate of $\Lambda$ and the fraction of recovered individuals from COVID-19, cholera, and both diseases with rates of $\mu_{1}, \mu_{2}$, and $\mu_{12}$, respectively, minus individuals newly exposed to COVID-19 and cholera. The rates of exposure to COVID-19 are governed by the time-dependent inputs $u_{1}(t)$ and $u_{2}(t)$, which correspond to the social measures taken during the course of the COVID-19 pandemic. While $u_{1}(t)$ refers to the rate of social distancing of asymptomatic carriers $(A), u_{2}(t)$ refers to the rate of quarantining of infected people $\left(I_{1}\right)$. On the other hand, the rate of incidence of cholera is governed by direct and indirect transmission rates. While human-to-human interaction forms the direct transmission $\alpha_{c}(t)$, the environmentto-human transmission forms the indirect transmission $\frac{\beta B}{K_{B}+B}$. The time-dependent input $u_{4}(t)$ corresponds to the fraction of susceptible individuals who have access to CWT for water purification.

Equation (2) describes individuals exposed to COVID-19. This epidemiological class includes a fraction of susceptible individuals minus individuals exposed during the latent period. Equation (3) describes individuals infected with COVID-19 but still asymptomatic or unconfirmed. The individuals of this epidemiological class come from the exposed class at a rate of $t_{\text {latent }}^{-1}$. This class will be left either by confirmation of COVID-19 infection or by direct recovery. Equation (4) describes confirmed infected individuals that have been tested. The screening level is measured by a time-dependent parameter $u_{3}(t)$. Thus, $u_{3}(t)$ is added to the other control variables $u_{1}(t), u_{2}(t), u_{3}(t)$ that will be used to verify the various measures taken during the COVID-19 epidemic. This epidemiological class will be left either for recovery at a rate of $\beta_{1}$, or for death at a rate of $\omega_{1}$, or for co-infection at a rate of $\delta_{1}$. Equation (5) describes individuals recovered from COVID-19 with or without symptoms. This class is increased due to the recovery of infected people in both classes 
from COVID-19 at rates of $\beta_{1}$ and $\rho$, respectively. On the other hand, this class is decreased due to infection with cholera or reinfection with COVID-19 at rate of $\lambda_{1}$ or $\mu_{1}$ respectively. Equation (6) describes individuals perished by COVID-19 at a rate of $\omega_{1}$. Equation (7) describes infected individuals with cholera after recovering from COVID19. This class is increased when the COVID-19 recovered individuals get infected with cholera at a rate of $\lambda_{1}$. It is reduced by recovering from COVID-19 and cholera respectively at a rate of $v_{1}$. Equation (8) describes individuals infected with both COVID-19 and cholera, either consecutively or simultaneously. The inputs to this class are either the infection of susceptible individuals or because a patient with one infection is infected with the other one at rates of $\alpha_{12}, \delta_{1}$, and $\delta_{2}$, respectively. It is decreased by the individuals' recovery at a rate of $v_{12}$ or individuals' death at rate of $\omega_{12}$. Equation (9) describes individuals recovered from both COVID-19 and cholera, either consecutively or simultaneously. It is increased when infected individuals recover from cholera, COVID-19, or both at rates of $v_{1}, v_{2}$, and $v_{12}$, respectively. It is decreased by natural death or becoming susceptible to both infections at a rate of $\mu_{12}$. Equation (10) describes individuals perished due to both COVID-19 and cholera at a rate of $\omega_{12}$. Equation (11) describes individuals infected with cholera. Cholera can be transmitted either directly through human's interaction at a rate of $\alpha_{c}(t)$ or by indirectly through bacteria ingestion with per capita contact rate of $\beta$. The probability of transmission from the environment is represented by $\frac{\beta B}{K_{B}+B}$, where $K_{B}$ is the level of pathogen concentration whereabouts half of all contacts with impure water produce infection and is related to bacteria concentration $B$ in the water. Therefore, the risk of infection increases as long as $B$ increases; and it decreases as $u_{4}(t)$ increases. People in this class have three options for leaving this class either to get dual infection at a rate of $\delta_{2}$, or to get recovered from cholera at a rate of $\beta_{2}$, or die due to cholera at a rate of $\omega_{2}$.

Equation (12) describes the environmental free bacteria. It is increased by the infected individuals at a rate of $\xi$, where humans can release bacteria into the open environment. This epidemiological class is increased by bacterial growth, which can be described by the logistic equation $r B(B-b)\left(1-\frac{B}{k}\right)$, where $r$ is the intrinsic growth, $k$ is the capacity of bacteria carrier, and $b$ is the Allee threshold when $\tau=0$. This epidemiological class is decreased by the death of bacteria with a mortality rate of $\tau$. Equation (13) describes individuals recovered from cholera at a rate of $\beta_{2}$. This epidemiological class is decreased by returning to being susceptible to both diseases or COVID-19 at rates of $\mu_{2}$ and $\lambda_{2}$, respectively. Equation (14) describes individuals dying due to cholera at a rate of $\omega_{2}$. Equation (15) describes individuals infected with COVID-19 after recovery from cholera at a rate of $\lambda_{2}$. The input of this class comes when the cholera recovered individuals are infected with COVID-19 at a rate of $\lambda_{2}$. Also, this class will be left by recovering from cholera and COVID-19 respectively at a rate of $v_{2}$. It is worth noting that the parameters in this work are classified into three categories. The first category includes time-varying inputs that reflect different rates of social distancing, quarantine, COVID-19 testing kit, and CWT for water purification. The second one includes values to be estimated from the real data obtained from Yemen such as transmission rate, recovery rate, and death rate. The third category includes literature-based values.

Furthermore, we can prove that the system is well defined as the classes' population are positive and bounded. 
Lemma 1 (Positivity of the solution) Let the initial conditions of model system (1)-(15) be nonnegative. Then the solutions of the proposed system are also nonnegative for all $t>0$

$$
\begin{array}{lcc}
S(t) \geq 0, & E(t) \geq 0, \quad A(t) \geq 0, \quad I_{1}(t) \geq 0, \quad R_{1}(t) \geq 0, \\
P_{1}(t) \geq 0, & I_{R 2}(t) \geq 0, \quad I_{2}(t) \geq 0, \quad R_{2}(t) \geq 0, \quad P_{2}(t) \geq 0, \\
I_{1 R}(t) \geq 0, \quad I_{12}(t) \geq 0, & R_{12}(t) \geq 0, \quad P_{12}(t) \geq 0 \quad \forall t>0 .
\end{array}
$$

Proof Let us take $t_{1}$ as

$$
\begin{aligned}
t_{1}= & \sup \left\{t>0: S(\tau)>0, E(\tau)>0, A(\tau)>0, I_{1}(\tau)>0, R_{1}(\tau)>0, P_{1}(\tau)>0,\right. \\
& I_{R 2}(\tau)>0, I_{2}(\tau)>0, R_{2}(\tau)>0, P_{2}(\tau)>0, I_{1 R}(\tau)>0, I_{12}(\tau)>0, \\
& \left.R_{12}(\tau)>0, P_{12}(\tau)>0 \forall \tau \in[0, t]\right\} .
\end{aligned}
$$

Consider $S(0) \geq 0, E(0) \geq 0, A(0) \geq 0, I_{1}(0) \geq 0, R_{1}(0) \geq 0, P_{1}(0) \geq 0, I_{R 2}(0) \geq 0, I_{2}(0) \geq$ $0, R_{2}(0) \geq 0, P_{2}(0) \geq 0, I_{1 R}(0) \geq 0, I_{12}(0) \geq 0, R_{12}(0) \geq 0, P_{12}(0) \geq 0$.

Now, let us take Eq. (1) as an example:

$$
\begin{aligned}
\frac{d S(t)}{d t}= & -\left(\frac{\left(1-u_{1}(t)\right)}{N} A(t)+\frac{\left(1-u_{2}(t)\right)}{N} I_{1}(t)+\alpha_{c}(t) I_{2}(t)\right. \\
& \left.+\frac{\beta B}{K_{B}+B}\left(1-u_{4}(t)\right)+\alpha_{12}\right) S(t)+\mu_{1} R_{1}(t)+\mu_{2} R_{2}(t)+\mu_{12} R_{12}(t)+\Lambda .
\end{aligned}
$$

For simplicity, we are assuming that

$$
f_{4}(s)=\left(\frac{\left(1-u_{1}(t)\right)}{N} A(t)+\frac{\left(1-u_{2}(t)\right)}{N} I_{1}(t)+\alpha_{c}(t) I_{2}(t)+\frac{\beta B}{K_{B}+B}\left(1-u_{4}(t)\right)+\alpha_{12}\right) .
$$

Thus, $\frac{d S(t)}{d t}+f_{4}(s) S(t)=\mu_{1} R_{1}(t)+\mu_{2} R_{2}(t)+\mu_{12} R_{12}(t)+\Lambda$.

This leads to

$$
\frac{d}{d t}\left(S(t) \exp \left(\int_{0}^{t_{1}} f_{4}(s) d s\right)\right)=\left(\mu_{1} R_{1}(t)+\mu_{2} R_{2}(t)+\mu_{12} R_{12}(t)+\Lambda\right) \exp \left(\int_{0}^{t_{1}} f_{4}(s) d s\right)
$$

Hence, we get on

$$
S(t)=\exp \left(-\int_{0}^{t_{1}} f_{4}(s) d s\right)\left[\int_{0}^{t_{1}}\left(\mu_{1} R_{1}(t)+\mu_{2} R_{2}(t)+\mu_{12} R_{12}(t)+\Lambda\right) \exp \left(\int_{0}^{t_{1}} f_{4}(s) d s\right)\right] .
$$

This proves that $S(t)>0$ for all $t>0$. In the same manner, we can prove that for all fifteen epidemiological classes.

Lemma 2 The solutions of system (1)-(15) are bounded.

In the proposed system (1)-(15), we have two different populations, the human and the bacteria population. 
Using the fact that the total human population at time $t$ is $X(t)$ and the reduced system of system (1)-(15) except (12), we obtain:

$$
\frac{d X(t)}{d t}=\Lambda-\xi I_{2}(t) \quad \Rightarrow \quad \frac{d X(t)}{d t} \leq \Lambda .
$$

By integrating both sides of the above equation, we have

$$
\Rightarrow X(t) \leq \Lambda+X_{0}
$$

Since each human epidemiological class is less than $X(t)$, all human classes are bounded. Hence all the solutions of system (1)-(15) related to the human and that initiating in $\left\{\mathbb{R}_{+}^{14} \backslash 0\right\}$ are confined in the region

$$
\begin{aligned}
\Omega= & \left\{\left(S(t), E(t), A(t), I_{1}(t), R_{1}(t), P_{1}(t), I_{R 2}(t), I_{2}(t), R_{2}(t), P_{2}(t),\right.\right. \\
& \left.\left.I_{1 R}(t), I_{12}(t), R_{12}(t), P_{12}(t)\right) \in \mathbb{R}_{+}^{14}: X<\Lambda+\varepsilon, \mid \varepsilon>0, t \rightarrow \infty\right\} .
\end{aligned}
$$

Now, we have some cases for the bacteria class $B(t)$.

From (12), we have

$$
\frac{d B(t)}{d t}=r B(B-b)\left(1-\frac{B}{k}\right)-\tau B+\xi I_{2}(t) .
$$

Since $I_{2}(t) \leq \Lambda+\varepsilon$ for all $t$, we obtain

$$
\frac{d B(t)}{d t} \leq r B(B-b)\left(1-\frac{B}{k}\right)-\tau B+\xi(\Lambda+\varepsilon) .
$$

In the absence of an infected case [11], we set

$$
f(B)=r B(B-b)\left(1-\frac{B}{k}\right)-\tau B
$$

If

$$
\tau \geq \frac{r(b-k)^{2}}{4 k}
$$

then $f(B)=0 \Leftrightarrow B=0 ; \lim _{B \rightarrow \infty} f(B)=-\infty$. In this case, the bacteria will become extinct.

If

$$
\tau<\frac{r(b-k)^{2}}{4 k}
$$

then we have two positive constants $a_{1}>b$ and $a_{2}>k$ given by

$$
a_{1}=\frac{r(b+k)-\sqrt{r^{2}(b-k)^{2}-4 k r \tau}}{2 r} \text { and } a_{2}=\frac{r(b+k)+\sqrt{r^{2}(b-k)^{2}-4 k r \tau}}{2 r}
$$

such that: 
- if $B(t)<a_{1}$, then the bacteria will become extinct;

- if $a_{1}<B(t)<a_{2}$, then the bacteria will exponentially increase up to $a_{2}$

- if $B(t)>a_{2}$, then the bacteria will exponentially decrease up to $a_{2}$,

where $a_{1}$ is the Allee threshold and $a_{2}$ is the carrying capacity. Hence Lemma 2.

Thus, for system (1)-(15), the population classes are positive and bounded. Hence, system (1)-(15) is well defined.

\section{Parameter estimation}

Based on the real data collected from Yemen (January 1, 2020 to May 30, 2020), this work aims to study the outbreaks of COVID-19 and cholera in the same country. The COVID19 data was obtained from the Center for Systems Science and Engineering (CSSE) at John Hopkins University (https://github.com/CSSEGISandData/COVID-19). The cholera data was obtained from the WHO and some data was taken from the health officials in Yemen. While the cholera data was taken weekly, that of COVID-19 was taken daily. Then the weekly data for both diseases was considered. Since the simultaneous data is required to compare and to determine the optimal policy, the data was standardized into weekly as that is more accurate than converting it into daily data.

To estimate the parameters, we used the least-squares regression approach, where we minimized the mean squared error (MSE) between prediction states and their available observation values derived from Yemen data according to Eq. (16):

$$
\min E_{0}=\sum_{l=0}^{N} w_{l}\left\|x_{l}-\hat{x}_{l}(t)\right\|^{2},
$$

in which $w_{l}$ are normalization weights and $x_{l}$ and $\hat{x}_{l}$ represent the number of each epidemiological class and its predictions, respectively. Pyomo optimization modelling implemented in Python is employed to solve the least-squares regression problem. The orthogonal collocation method is used to discretize the dynamic differential equations system (1)-(15) taking into consideration the time domain which consists of weekly finite elements. The means of the estimated parameters are the following: $E_{0}=0.28499, \mu_{1}=0$, $\mu_{2}=0.00030, \mu_{12}=0, \beta=0.02325, \alpha_{c}(t)=0.25, \delta_{1}=0, \delta_{2}=0, \beta_{1}=0.005, \beta_{2}=1.5, \lambda_{1}=0$, $\lambda_{2}=0, v_{1}=0, v_{2}=0, v_{12}=0, \omega_{1}=0.04470, \omega_{12}=0, \omega_{2}=0.0002, \xi=40, \tau=0.333$, and $\alpha_{12}=0$. These estimated values of the parameters were obtained from historical data for both infections in Yemen (January 1, 2020 to May 30, 2020), and they will be used to solve the optimal control model in the next section. As the confirmed infected cases of co-infection simultaneously or consecutively in Yemen are still nonexistent, the parameter values of the dual infection equal zero. By comparing the estimated values of the parameters with the parameters estimated in [17], we find that the death rate due to COVID-19 in Yemen is so high at the rate of 0.0447, while the death rate due to COVID19 in Germany is lower than other at the rate of 0.0024. According to [17] in some other countries, as the USA, the death rate is 0.0044 , in Italy it is 0.010619 , and in Spain it is 0.011871. Regarding the recovered cases, Germany occupies the first position at the rate of (0.046838), then comes Spain at the rate of 0.040129. In the third place comes Italy at the rate of 0.016644 . The USA occupies the fourth position at the rate of 0.007467 , and Yemen comes in the last position at the rate 0.005. According to [11] in 2017, while the death and recovery rates of cholera were 0.0003 and 1.4, respectively, in this study there 


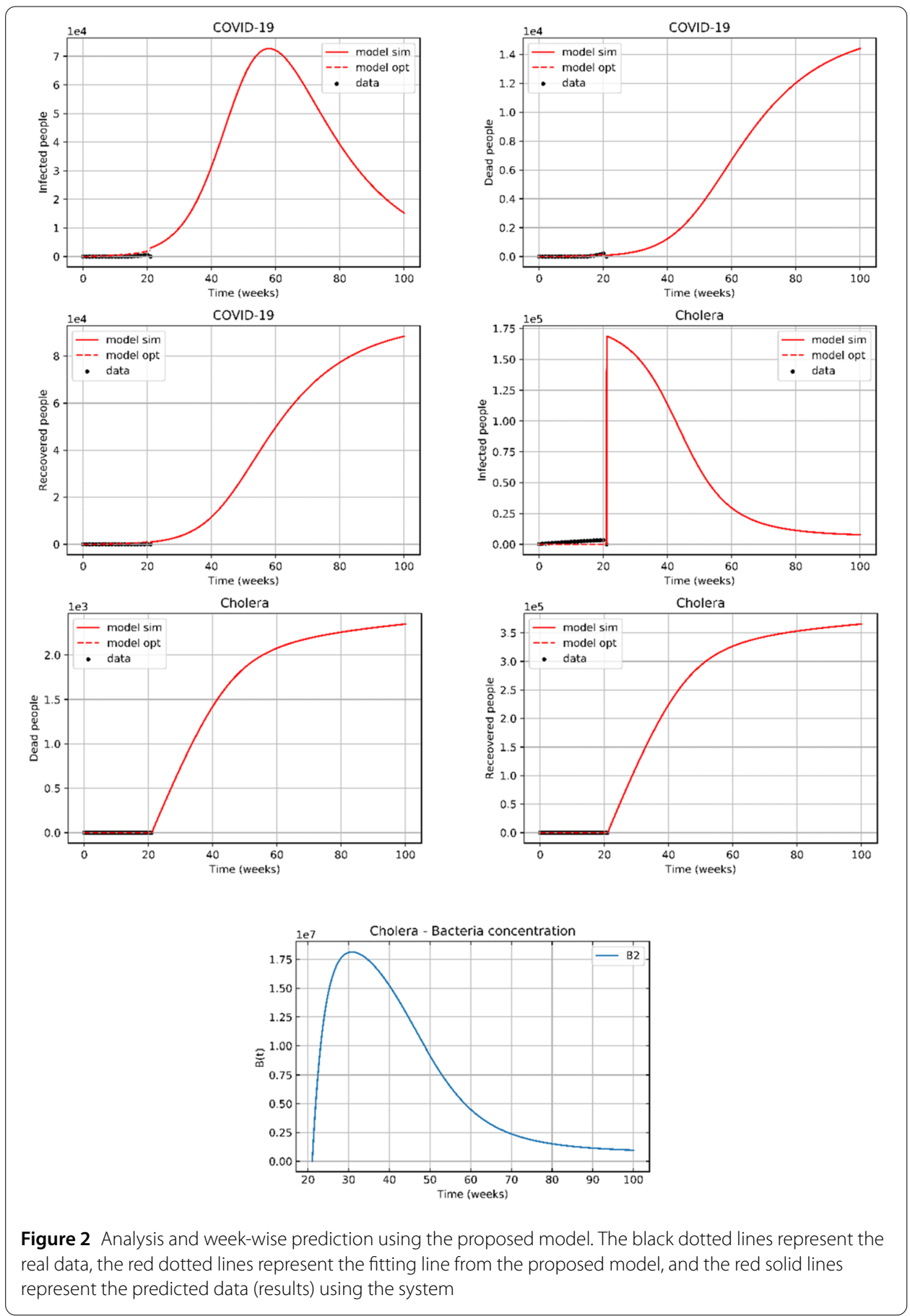

is a slight improvement as the death and recovery rates of cholera are 0.0002 and 1.5 , respectively.

Furthermore, the predicted values of the infected, recovered, and deaths of both diseases were obtained, as illustrated in Fig. 2. Note that the mortality rate due to COVID-19 is very high compared to the mortality rate due to cholera even though the number of infected individuals with cholera is much higher than the number of infected individuals with COVID-19. 


\section{Optimal control problem}

Both epidemics are optimally controlled by minimizing the total number of infected individuals and this is done by controlling time-dynamic parameters appropriate for Yemen. The control policy that we propose aims at minimizing the number of exposed individuals to COVID-19 by controlling the parameters related to lockdown, social distancing, and the number of tests. The number of infected individuals with cholera can be minimized by increasing the distribution of CWTs for water purification for all susceptible individuals. This helps to minimize the number of infected individuals with COVID-19 or cholera or co-infections.

In our model, we consider four control functions $u_{1}(t), u_{2}(t), u_{3}(t)$, and $u_{4}(t)$. The first $u_{1}(t)$ represents the social distancing rate. This is an important parameter because it can be applied in Yemen by requiring people to distance socially, to wear masks, and to improve their personal hygiene. Therefore, $u_{1}(t)$ assists us in examining its impact on reducing the COVID-19 outbreak. The second control function $u_{2}(t)$ represents the quarantine and isolation rates. Most people in Yemen live on daily wages, which means that it is difficult to implement a complete lockdown on cities. Hence, only small values of this parameter are used. $u_{2}(t)$ allows us to investigate its impact on curbing the COVID-19 outbreak. The third control function $u_{3}(t)$ represents the number of COVID-19 test kits. By increasing the number of test kits, infected individuals can be discovered quickly, which means that they can be isolated and treated promptly. Therefore, $u_{3}(t)$ allows us to investigate its impact on mitigating the spread of the COVID-19 epidemic. The last control function $u_{4}(t)$ represents the fraction of susceptible individuals who can get CWTs for water purification. By increasing $u_{4}(t)$, the water will be purer and the vector cholera and other diseases will be eliminated. Therefore, $u_{4}(t)$ allows us to investigate its impact on curbing the cholera outbreak.

The objective function of the proposed model is to minimize the total number of new infected cases with both diseases as well as the cost associated with each control over a particular period of time. The objective function is formulated as follows:

$$
\begin{aligned}
& \min _{u_{i}(t), i=1, . ., 4} \int_{t_{0}}^{t_{f}} z_{1} I_{1}(t)+z_{2} I_{2}(t)+z_{3} I_{12}(t) \\
& \quad+D_{1}\left(u_{1}(t)\right)^{2}+D_{2}\left(u_{2}(t)\right)^{2}+D_{3}\left(u_{3}(t)\right)^{2} \\
& (1-15), \\
& \max _{t}\left(I_{l}(t)\right) \leq I_{l}^{\text {peak }}, \quad l=1,2, \\
& u_{1}(t) \in[0.05,0.5], \\
& u_{2}(t) \in[0.01,0.3], \\
& u_{3}(t) \in[0.1,0.3], \\
& u_{4}(t) \in[0,1] .
\end{aligned}
$$$$
+D_{1}\left(u_{1}(t)\right)^{2}+D_{2}\left(u_{2}(t)\right)^{2}+D_{3}\left(u_{3}(t)\right)^{2}+D_{4}\left(u_{4}(t)\right)^{2},
$$

The objective function in Eq. (17) minimizes the number of individuals infected with COVID-19, cholera, and both. It also minimizes the total costs associated with the control interventions. The constraints of the optimal control model are incorporated in Eqs. (1)(15). The bound of the time-dependent control variables and the infected individuals for 
each infection must be under the estimated peaks for each infection. Constraint (18) determines the limit of the peak size for each epidemic. Constraints (19)-(22) determine the ranges of the time-dynamic parameters, where $z_{1}, z_{2}, z_{3}, D_{1}, D_{2}, D_{3}$, and $D_{4}$ are weight coefficients. The optimal control model is assumed during the full-time horizon $\left[t_{0}, t_{f}\right]$, where $t_{f}$ is 100 weeks.

Therefore, the solution of the optimal control model is $\left(u_{1}^{*}(t), u_{2}^{*}(t), u_{3}^{*}(t), u_{4}^{*}(t)\right)$ in which

$$
J\left(u_{1}^{*}(t), u_{2}^{*}(t), u_{3}^{*}(t), u_{4}^{*}(t)\right)=\min \left\{J\left(u_{1}(t), u_{2}(t), u_{3}(t), u_{4}(t)\right) \mid u_{i}(t) \in \Psi, i=1, \ldots 4\right\},
$$

where $\Psi=\left(u_{1}(t), u_{2}(t), u_{3}(t), u_{4}(t)\right)$ and $0 \leq u_{1}(t) \leq 0.5,0 \leq u_{2}(t) \leq 0.3,0 \leq u_{3}(t) \leq 0.3$, $0 \leq u_{4}(t) \leq 1$

Pontryagin's maximum principle is an indirect method to solve the optimal control model through derivation of the Hamiltonian function and defining the necessary conditions for the optimal control of the cholera-COVID-19 co-infection model. The Hamiltonian $H$ is defined as follows:

$$
\begin{aligned}
H= & z_{1} I_{1}(t)+z_{2} I_{2}(t)+z_{3} I_{12}(t)+D_{1}\left(u_{1}(t)\right)^{2}+D_{2}\left(u_{2}(t)\right)^{2}+D_{3}\left(u_{3}(t)\right)^{2} \\
& +D_{4}\left(u_{4}(t)\right)^{2}+M_{S}\left(\mu_{1} R_{1}(t)+\mu_{2} R_{2}(t)+\mu_{12} R_{12}(t)+\Lambda-\left(\frac{\left(1-u_{1}(t)\right)}{N} A(t)\right.\right. \\
& \left.\left.+\frac{\left(1-u_{2}(t)\right)}{N} I_{1}(t)+\alpha_{c}(t) I_{2}(t)+\frac{\beta B}{K_{B}+B}\left(1-u_{4}(t)\right)+\alpha_{12}\right) S(t)\right) \\
& +M_{E}\left(\left(\frac{\left(1-u_{1}(t)\right)}{N} A(t)+\frac{\left(1-u_{2}(t)\right)}{N} I_{1}(t)\right) S(t)-t_{\text {latent }}^{-1} E(t)\right) \\
& +M_{A}\left(t_{\text {latent }}^{-1} E(t)-\left(u_{3}(t)+\rho\right) A(t)\right)+M_{I_{1}}\left(u_{3}(t) A(t)-\left(\delta_{1}+\beta_{1}+\omega_{1}\right) I_{1}(t)\right) \\
& +M_{R_{1}}\left(\rho A(t)+\beta_{1} I_{1}(t)-\left(\mu_{1}+\lambda_{1}\right) R_{1}(t)\right)+M_{P_{1}}\left(\omega_{1} I_{1}(t)\right)+M_{I_{R 2}}\left(\lambda_{1} R_{1}(t)\right. \\
& \left.-v_{1} I_{R 2}(t)\right)+M_{I_{12}}\left(\alpha_{12} S(t)+\delta_{1} I_{1}(t)+\delta_{2} I_{2}(t)-\left(v_{12}+\omega_{12}\right) I_{12}(t)\right) \\
& +M_{R_{12}}\left(v_{1} I_{R 2}(t)+v_{2} I_{1 R}(t)+v_{12} I_{12}(t)-\mu_{12} R_{12}(t)\right)+M_{P_{12}}\left(\omega_{12} I_{12}(t)\right) \\
& +M_{I_{2}}\left(\left(\alpha_{c}(t) I_{2}(t)+\frac{\beta B}{K_{B}+B}\left(1-u_{4}(t)\right)\right) S(t)-\left(\delta_{2}+\beta_{2}+\omega_{2}+\xi\right) I_{2}(t)\right) \\
& +M_{B}\left(r B(B-b)\left(1-\frac{B}{k}\right)-\tau B+\xi I_{2}(t)\right)+M_{R_{2}}\left(\beta_{2} I_{2}(t)-\left(\mu_{2}+\lambda_{2}\right) R_{2}(t)\right) \\
& +M_{P_{2}}\left(\omega_{2} I_{2}(t)\right)+M_{I_{1 R}}\left(\lambda_{2} R_{2}(t)-v_{2} I_{1 R}(t)\right),
\end{aligned}
$$

where $M_{S}, M_{E}, M_{A}, M_{I_{1}}, M_{R_{1}}, M_{P_{1}}, M_{I_{R 2}}, M_{I_{12}}, M_{R_{12}}, M_{P_{12}}, M_{I_{2}}, M_{B}, M_{R_{2}}, M_{P_{2}}$, and $M_{I_{1 R}}$ are adjoint variables or co-state variables.

Theorem Given the optimal control $u_{1}^{*}(t), u_{2}^{*}(t), u_{3}^{*}(t), u_{4}^{*}(t)$ and solutions $S, E, A, I_{1}, R_{1}$, $P_{1}, I_{R 2}, I_{12}, R_{12}, P_{12}, I_{2}, B, R_{2}, P_{2}$, and $I_{1 R}$ of the corresponding state system (1)-(15) that minimize $J\left(u_{1}(t), u_{2}(t), u_{3}(t), u_{4}(t)\right)$ over $\Psi$. There exist adjoint variables $M_{S}, M_{E}, M_{A}, M_{I_{1}}$, $M_{R_{1}}, M_{P_{1}}, M_{I_{R 2}}, M_{I_{12}}, M_{R_{12}}, M_{P_{12}}, M_{I_{2}}, M_{B}, M_{R_{2}}, M_{P_{2}}$, and $M_{I_{1 R}}$ such that

$$
\frac{-d M_{l}}{d t}=\frac{\partial H}{\partial l},
$$


where $l=S, E, A, I_{1}, R_{1}, P_{1}, I_{R 2}, I_{12}, R_{12}, P_{12}, I_{2}, B, R_{2}, P_{2}$, and $I_{1 R}$ with the transversality conditions

$$
\begin{aligned}
M_{S}\left(t_{f}\right) & =M_{E}\left(t_{f}\right)=M_{A}\left(t_{f}\right)=M_{I_{1}}\left(t_{f}\right)=M_{R_{1}}\left(t_{f}\right)=M_{P_{1}}\left(t_{f}\right)=M_{I_{R 2}}\left(t_{f}\right)=M_{I_{12}}\left(t_{f}\right) \\
& =M_{R_{12}}\left(t_{f}\right)=M_{P_{12}}\left(t_{f}\right)=M_{I_{2}}\left(t_{f}\right)=M_{B}\left(t_{f}\right)=M_{R_{2}}\left(t_{f}\right)=M_{P_{2}}\left(t_{f}\right)=M_{I_{1 R}}\left(t_{f}\right)=0
\end{aligned}
$$

and

$$
\begin{aligned}
& u_{1}^{*}(t)=\min \left\{1, \max \left(0, \frac{\left(M_{E}-M_{S}\right) A(t) S(t)}{2 D_{1} N}\right)\right\}, \\
& u_{2}^{*}(t)=\min \left\{1, \max \left(0, \frac{\left(M_{E}-M_{S}\right) I_{1}(t) S(t)}{2 D_{2} N}\right)\right\}, \\
& u_{3}^{*}(t)=\min \left\{1, \max \left(0, \frac{\left(M_{A}-M_{I_{1}}\right) A(t)}{2 D_{3}}\right)\right\}, \\
& u_{4}^{*}(t)=\min \left\{1, \max \left(0, \frac{\left(M_{I_{2}}-M_{S}\right) \beta B S(t)}{2 D_{4}\left(K_{B}+B\right)}\right)\right\} .
\end{aligned}
$$

The proof of this theorem is in Appendix A.

\section{Numerical simulations}

In this section, we investigate and analyze the impact of the four dynamic control measures and the peak limits, which will determine the best policy for curbing the rapid spread of cholera and COVID-19 in Yemen in 2020. The policy approach is based on monotonous declines over time for quarantine, social separation, and monotonic increases for both the number of COVID-19 tests and the number of CWTs for water purification. Pyomo optimization modelling in Python was used to solve the optimal control model (16)-(21). The orthogonal collocation method is again used to discretize the dynamic differential equations system (1)-(15) taking into consideration the time domain which consists of weekly finite elements. Parameter values obtained from the solution of the parameters estimation problem in Sect. 3 are used for solving the optimal control model. Moreover, the other parameters and initial conditions in the optimal control model are fixed as indicated in Table 1 and Table 2 for all the following simulations. Table 3 illustrates the range of the time-dynamic functions for each policy. The following policies are discussed.

Policy 1: (No control) Baseline

In this policy, all coefficients of the time-dynamic variables in the objective function and the constraints are assumed to be equal to zero. In addition, the limit of the peaks for both outbreaks is equal to the maximum peaks indicated in Table 3.

Table 3 Ranges of control parameters and values of limit peaks for each policy

\begin{tabular}{lllc}
\hline Policy & $\left(\left[\alpha_{a}^{\prime}, \alpha_{a}^{u}\right],\left[\alpha_{i}^{\prime}, \alpha_{i}^{u}\right],\left[\kappa^{\prime}, \kappa^{u}\right],\left[u^{\prime}, u^{u}\right]\right)$ & $\left(l_{1}^{\text {peak }}, P_{2}^{\text {peak }}\right)$ & The associated cost \\
\hline No control & $([0,0],[0,0],[0,0],[0,0])$ & $\left(10^{5}, 5 \times 10^{5}\right)$ & - \\
Med control & $([0.2,0.4],[0.1,0.2],[0.2,0.25],[0.25,0.75])$ & $\left(10^{4}, 5 \times 10^{5}\right)$ & 4.5349228133 \\
Max control & $([0.4,0.5],[0.25,0.3],[0.25,0.3],[0.75,1])$ & $\left(10^{4}, 5 \times 10^{5}\right)$ & 2.8707575932 \\
Mix control & $([0.2,0.4],[0,0.1],[0.2,0.3],[0.7,0.9])$ & $\left(10^{4}, 5 \times 10^{5}\right)$ & 1.9432928849 \\
Min peaks & $([0.4,0.5],[0.1,0.2],[0.15,0.2],[025,0.95])$ & $\left(10^{4}, 5 \times 10^{5}\right)$ & 0.16289408246 \\
Max peaks & $([0.4,0.5],[0.1,0.2],[0.15,0.2],[0.25,0.95])$ & $\left(10^{5}, 5 \times 10^{5}\right)$ & 13.678641590 \\
\hline
\end{tabular}


The obtained results of the numerical simulations show that this combination is not good to curb both outbreaks. From the panels of the infected individuals with both epidemics in Fig. 4, Appendix B, we note that the peaks infected individuals with COVID-19 and cholera are high and their curves take too long to come down. Similarly, the panel of the bacteria population shows that free bacteria growth is the highest in growth and the slowest to decline over time.

Policy 2: (Med control) Sufficient resources for both CWT distribution and test kits with average social distancing and quarantining

In this policy, we assume that test kits are in the range of $20 \%-25 \%$ and are sufficient for the number of individuals susceptible to COVID-19. We also assume that CWTs are distributed to $25 \%-75 \%$ of the individuals susceptible to cholera. Moreover, the average values of the proportions of social distancing and quarantining are considered. Quarantining is applied only with a ratio of less than $20 \%$ and social distancing is applied with a ratio of less than $40 \%$.

The results are shown in Fig. 5 in Appendix B. From the control time-dynamic functions panel, we observe that all time-dynamic functions have changed dynamically in the defined range referred to in Table 3 . However, the results for this policy improve compared to those for the baseline policy, especially in terms of the number of infected individuals over time for both epidemics, the epidemic periods, and the bacteria growth.

Policy 3: (Max control) Abundant resources for both CWT distribution and test kits with strict social distancing and quarantining

In this strategy, we assume that almost all individuals susceptible to cholera have access to pure water and that test kits are available for almost all individuals susceptible to COVID-19. Moreover, strict rules for social distancing and quarantining are assumed in this policy.

The results are shown in Fig. 6 in Appendix B. From the control time-dynamic functions panel, we observe that while $u_{4}(t)$ and $u_{3}(t)$ increase monotonically, $u_{1}(t)$ and $u_{2}(t)$ decrease monotonically. Moreover, the fitted lines almost match the real data for both infections. This policy can affect the curve peaks for both epidemics and can end the epidemic period. Also, this policy can inhibit the rapid growth of bacteria.

Policy 4: (Mix control) Abundant resources for CWT distribution and sufficient resources for test kits with average social distancing and low quarantining

In this policy, we assume that almost all individuals susceptible to cholera have access to pure water and that test kits are available for a sufficient number of individuals susceptible to COVID-19. In addition, we assume that average social distancing is applied with less quarantining. From Fig. 7, Appendix B, we note that there are slight changes in the results of this policy compared to those of the Max control policy in terms of the number of cases for each epidemiological class, the peaks, and the speed of ending the epidemic. The results indicate the importance of increasing $u_{3}(t)$ and $u_{4}(t)$ and imposing social distancing $u_{1}(t)$, and in return the lack of complete social quarantine $u_{2}(t)$, since it has negative economic effects.

Policy 5: Min peaks

We assumed in all the aforementioned policies that the peak of COVID-19 does not exceed 100,000 and that the peak of cholera does not exceed 300,000. In this policy, we change the peak of COVID-19 to be less than 10,000 and the peak of cholera be less than 
100,000. The ranges of the time-dynamic measures used in this policy are shown in Table 3.

We can see from the results in Fig. 8, Appendix B that the number of infected individuals for each epidemic is not high, while maintaining the same pattern of results with the previous policies.

Policy 6: Max peaks

In this scenario, we investigate the effect of limit values of peaks on the numbers of each epidemic class by increasing the limits of the peak values as indicated in Table 3.

In Fig. 9, Appendix B a significant increase in all the numbers of COVID-19 epidemic classes and a slight increase in the cholera epidemic classes are noticed.

We summarize the range of the control parameters for each policy, the values of the limit peaks, and the obtained cost of all simulations in Table 3 . We can observe clearly that policy 5 (Min peaks) has minimum cost with an objective function value of 0.16289408246 .

Figure 3 shows the comparison of the simulation results of the different policies for all the epidemiological classes. Having a glance at the panel of infected individuals with COVID-19, it is clear that policy 3 (Max control) leads to a flatter epidemic curve and the lowering in the number of infected individuals. In the second rank comes policy 4 (Mix control), which corresponds to distributing CWTs to all individuals susceptible to cholera, providing a sufficient number of test kits with a reasonable commitment to social distancing, and quarantining only for the infected individuals with COVID-19. Moreover, policy 1 (No control) leads to the highest peak size.

On the other side, having a look at the panel of perished individuals due to COVID-19, it is shown that policy 1 (No control) is the worst among all the other policies. Nevertheless, policy 3 with Max control leads to the lowest number of people lost due to COVID-19. The panel of recovered individuals from COVID-19 shows that policy 1 (No control) leads to the highest number of recovered individuals as compared to other policies. This was expected due to the increase in the number of infected individuals with COVID-19.

The panel of the infected individuals with cholera shows that policy 3 (Max control) is the best to reduce the number of infected individuals as well as the fastest in ending the epidemic. Policy 1 (Baseline) is the worst in terms of the number of infected individuals having the highest peak size. In addition, it has also the longest epidemic period. The panel of the perished individuals due to cholera shows that while the first and second policies (Baseline and Med control) lead to the highest numbers, the third and fourth policies (Max and Mix control) lead to the lowest numbers. Conversely, the panel of the recovered individuals from cholera shows that while the first and second policies (Baseline and Med control) lead to the highest number of the recovered individuals, the third and fourth policies (Max and Mix control) lead to the lowest number of the recovered individuals due to the large numbers of infected individuals with cholera.

The panel of the bacteria population shows that policy 3 (Max control) has the lowest peak and leads to the rapid decrease of the bacterial population growth. This contributes to reducing the number of individuals susceptible to cholera. Policy 4 (Mix control) comes in the second rank in terms of the peak and the return of the bacterial population curve. Our results show that while some policies are efficient in reducing the number of individuals infected with COVID-19, others are efficient in controlling the number of individuals infected with cholera (the ranks of the policies according to epidemiological class are shown in Table 4, Appendix C). 


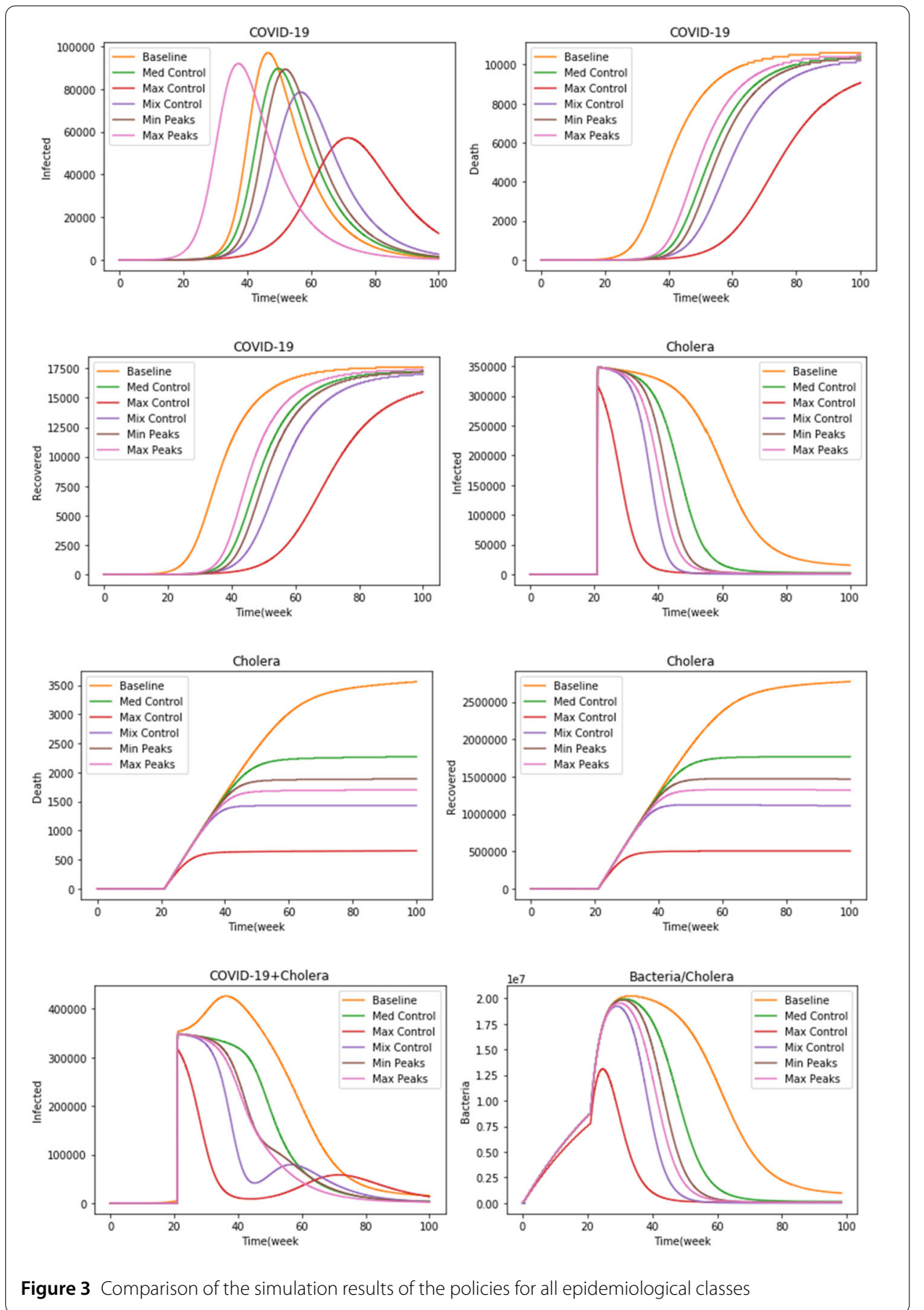

To determine the optimal policy that can be applied in Yemen, we can combine the coinfected cases of each policy. The results are shown in the panel of the infected individuals with COVID-19 + cholera. The third policy (Max control) is shown as the best among all the policies proposed to control and reduce the number of infected people with both epidemics. As applying a complete quarantine in a poor country like Yemen is not possible for its people earn their living on daily wage and lack awareness of the dangers of these epidemics, it is concluded that the most effective policy that can be carried out in Yemen is policy 4 (Mix control) since it seeks to increase the number of CWTs for water purification 
to almost all the individuals susceptible to cholera and to increase the number of test kits for the exposed individuals to COVID-19. Besides, reasonable social distancing has to be applied with quarantining for only infected cases.

\section{Conclusions}

In the present work, we formulated a compartmental cholera-COVID-19 co-infection model that describes the dynamics of transmission of COVID-19 and cholera in Yemen. Then, we estimated the parameters of the model and proposed an optimal control policy to minimize the number of individuals infected with both infections and to minimize the total cost associated with each control. Then, optimal control policies were investigated. Those include four control functions: social distancing, lockdown, the number of test kits to control the COVID-19 outbreak, and the number of susceptible individuals who can get $\mathrm{CWTs}$ for water purification. We investigated the advantages of each policy by comparing the results of the numerical simulations. We demonstrated that the optimal policy to be applied in Yemen is based on social distancing to protect susceptible individuals from the infections, increasing the number of test kits for infected individuals, and increasing the number of CWTs for water purification. For future work, dual COVID-19 with other infections can be considered and other time-dynamic functions for controlling the infection outbreaks can be added.

\section{Appendix A}

In this appendix, we prove the theorem in Sect. 4.

Proof By applying Pontryagin's maximum principle to the Hamiltonian $H$, we get the following adjoint systems:

$$
\begin{aligned}
\frac{d M_{S}}{d t}= & -\frac{\partial H}{\partial S} \\
= & \left(M_{S}-M_{E}\right)\left(\frac{\left(1-u_{1}(t)\right)}{N} A(t)+\frac{\left(1-u_{2}(t)\right)}{N} I_{1}(t)\right) \\
& +\left(M_{S}-M_{I_{2}}\right)\left(\alpha_{c}(t) I_{2}(t)+\frac{\beta B}{K_{B}+B}\left(1-u_{4}(t)\right)\right)+\alpha_{12}\left(M_{S}-M_{I_{12}}\right), \\
\frac{d M_{E}}{d t}= & -\frac{\partial H}{\partial E}=\left(M_{E}-M_{A}\right) t_{\text {latent }}^{-1}, \\
\frac{d M_{A}}{d t}= & -\frac{\partial H}{\partial A}=\left(M_{S}-M_{E}\right)\left(\frac{\left(1-u_{1}(t)\right)}{N}\right) S(t)+u_{3}(t)\left(M_{A}-M_{I_{1}}\right)+\rho\left(M_{A}-M_{R_{1}}\right), \\
\frac{d M_{I_{1}}}{d t}= & -\frac{\partial H}{\partial I_{1}} \\
= & -z_{1}+\left(M_{S}-M_{E}\right)\left(\frac{u_{2}(t) S(t)}{N}\right) \\
& +\beta_{1}\left(M_{I_{1}}-M_{R_{1}}\right)+\omega_{1}\left(M_{I_{1}}-M_{P_{1}}\right)+\delta_{1}\left(M_{I_{1}}-M_{I_{12}}\right), \\
\frac{d M_{R_{1}}}{d t}= & -\frac{\partial H}{\partial R_{1}}=\left(M_{R_{1}}-M_{S}\right) \mu_{1}+\left(M_{R_{1}}-M_{I_{22}}\right) \lambda_{1}, \\
\frac{d M_{P_{1}}}{d t}= & -\frac{\partial H}{\partial P_{1}}=0,
\end{aligned}
$$




$$
\begin{aligned}
\frac{d M_{I_{R 2}}}{d t}= & -\frac{\partial H}{\partial I_{R 2}}=\left(M_{I_{R 2}}-M_{R_{12}}\right) v_{1} \\
\frac{d M_{I_{12}}}{d t}= & -\frac{\partial H}{\partial I_{12}}=-z_{3}+\left(M_{I_{12}}-M_{R_{12}}\right) v_{12}+\left(M_{I_{12}}-M_{P_{12}}\right) \omega_{12} \\
\frac{d M_{R_{12}}}{d t}= & -\frac{\partial H}{\partial R_{12}}=\left(M_{R_{12}}-M_{S}\right) \mu_{12} \\
\frac{d M_{P_{12}}}{d t}= & \frac{\partial H}{\partial P_{12}}=0 \\
\frac{d M_{I_{2}}}{d t}= & -\frac{\partial H}{\partial I_{2}} \\
= & -z_{2}+\alpha_{c}(t) S(t)\left(M_{S}-M_{I_{2}}\right)+\beta_{2}\left(M_{I_{2}}-M_{R_{2}}\right)+\omega_{2}\left(M_{I_{2}}-M_{P_{2}}\right) \\
& +\delta_{2}\left(M_{I_{2}}-M_{I_{12}}\right)+\xi\left(M_{I_{2}}-M_{B}\right) \\
\frac{d M_{B}}{d t}= & -\frac{\partial H}{\partial B}=M_{B} \tau+M_{B}\left(\frac{2 B b}{K_{B}}-3 r B^{2}\right)+\left(M_{B}-M_{I_{2}}\right)\left(\frac{K_{B}}{\left(K_{B}+B\right)^{2}}\right)\left(1-u_{4}(t)\right) S(t) \\
\frac{d M_{R_{2}}}{d t}= & -\frac{\partial H}{\partial R_{2}}=\left(M_{R_{2}}-M_{S}\right) \mu_{2}+\left(M_{R_{1}}-M_{I_{1 R}}\right) \lambda_{2} \\
\frac{d M_{P_{2}}}{d t}= & \frac{\partial H}{\partial P_{2}}=0 \\
\frac{d M_{I_{1 R}}}{d t}= & -\frac{\partial H}{\partial I_{1 R}}=\left(M_{I_{1 R}}-M_{R_{12}}\right) v_{2} .
\end{aligned}
$$

We obtain the time-dependent function controls $u_{1}^{*}(t), u_{2}^{*}(t), u_{3}^{*}(t), u_{4}^{*}(t)$ when $\frac{\partial H}{\partial u_{1}(t)}=$ $\frac{\partial H}{\partial u_{2}(t)}=\frac{\partial H}{\partial u_{3}(t)}=\frac{\partial H}{\partial u_{4}(t)}=0$. Then, we obtain the following:

$$
\begin{aligned}
\frac{\partial H}{\partial u_{1}(t)} & =2 D_{1} u_{1}(t)+\frac{A(t) S(t)}{N}\left(M_{S}-M_{E}\right)=0, \\
\frac{\partial H}{\partial u_{2}(t)} & =2 D_{2} u_{2}(t)+M_{S} \frac{I_{1}(t) S(t)}{N}-M_{E} \frac{I_{1}(t) S(t)}{N}=0, \\
\frac{\partial H}{\partial u_{3}(t)} & =2 D_{3} u_{3}(t)-M_{A} A(t)+M_{I_{1}} A(t)=0, \\
\frac{\partial H}{\partial u_{4}(t)} & =2 D_{4} u_{4}(t)+M_{S} \frac{\beta B S(t)}{K_{B}+B}-M_{I_{2}} \frac{\beta B S(t)}{K_{B}+B}=0 .
\end{aligned}
$$

Hence, we obtain

$$
\begin{aligned}
& u_{1}^{*}(t)=\frac{\left(M_{E}-M_{S}\right) A(t) S(t)}{2 D_{1} N}, \\
& u_{2}^{*}(t)=\frac{\left(M_{E}-M_{S}\right) I_{1}(t) S(t)}{2 D_{2} N}, \\
& u_{3}^{*}(t)=\frac{\left(M_{A}-M_{I_{1}}\right) A(t)}{2 D_{3}}, \\
& u_{4}^{*}(t)=\frac{\left(M_{I_{2}}-M_{S}\right) \beta B S(t)}{2 D_{4}\left(K_{B}+B\right)} .
\end{aligned}
$$


By the standard control arguments involving the bounds on the controls, we conclude

$$
\begin{aligned}
& u_{1}^{*}(t)= \begin{cases}0 & \text { if } \frac{\left(M_{E}-M_{S}\right) A(t) S(t)}{2 D_{1} N} \leq 0, \\
\frac{\left(M_{E}-M_{S}\right) A(t) S(t)}{2 D_{1} N} & \text { if } 0<\frac{\left(M_{E}-M_{S}\right) A(t) S(t)}{2 D_{1} N}<1, \\
1 & \text { if } \frac{\left(M_{E}-M_{S}\right) A(t) S(t)}{2 D_{1} N} \geq 1,\end{cases} \\
& u_{2}^{*}(t)= \begin{cases}0 & \text { if } \frac{\left(M_{E}-M_{S}\right) I_{1}(t) S(t)}{2 D_{2} N} \leq 0, \\
\frac{\left(M_{E}-M_{S}\right) I_{1}(t) S(t)}{2 D_{2} N} & \text { if } 0<\frac{\left(M_{E}-M_{S}\right) I_{1}(t) S(t)}{2 D_{2} N}<1, \\
1 & \text { if } \frac{\left(M_{E}-M_{S}\right) I_{1}(t) S(t)}{2 D_{2} N} \geq 1,\end{cases} \\
& u_{3}^{*}(t)= \begin{cases}0 & \text { if } \frac{\left(M_{A}-M_{I_{1}}\right) A(t)}{2 D_{3}} \leq 0, \\
\frac{\left(M_{A}-M_{I_{1}}\right) A(t)}{2 D_{3}} & \text { if } 0<\frac{\left(M_{A}-M_{I_{1}}\right) A(t)}{2 D_{3}}<1, \\
1 & \text { if } \frac{\left(M_{A}-M_{I_{1}}\right) A(t)}{2 D_{3}} \geq 1,\end{cases} \\
& u_{4}^{*}(t)= \begin{cases}0 & \text { if } \frac{\left(M_{I_{2}}-M_{S}\right) \beta B S(t)}{2 D_{4}\left(K_{B}+B\right)} \leq 0, \\
\frac{\left(M_{I_{2}}-M_{S}\right) \beta B S(t)}{2 D_{4}\left(K_{B}+B\right)} & \text { if } 0<\frac{\left(M_{I_{2}}-M_{S}\right) \beta B S(t)}{2 D_{4}\left(K_{B}+B\right)}<1, \\
1 & \text { if } \frac{\left(M_{I_{2}}-M_{S}\right) \beta B S(t)}{2 D_{4}\left(K_{B}+B\right)} \geq 1 .\end{cases}
\end{aligned}
$$

\section{Appendix B}

In this appendix, we show the graphical results of all of the policies investigated in the study. 
Policy 1: (No control) Baseline
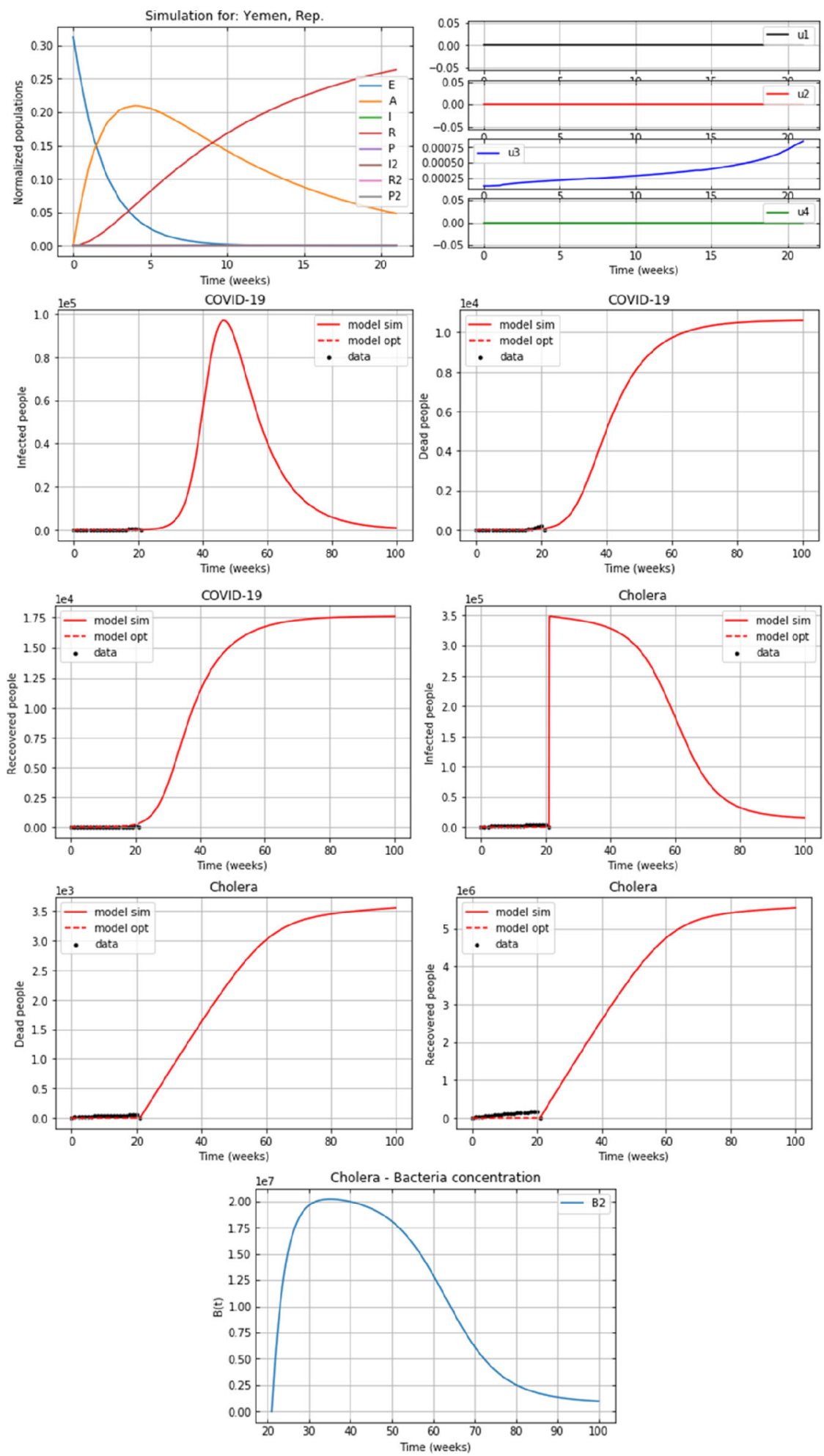

Figure 4 Results for policy 1 
Policy 2 (Med control)
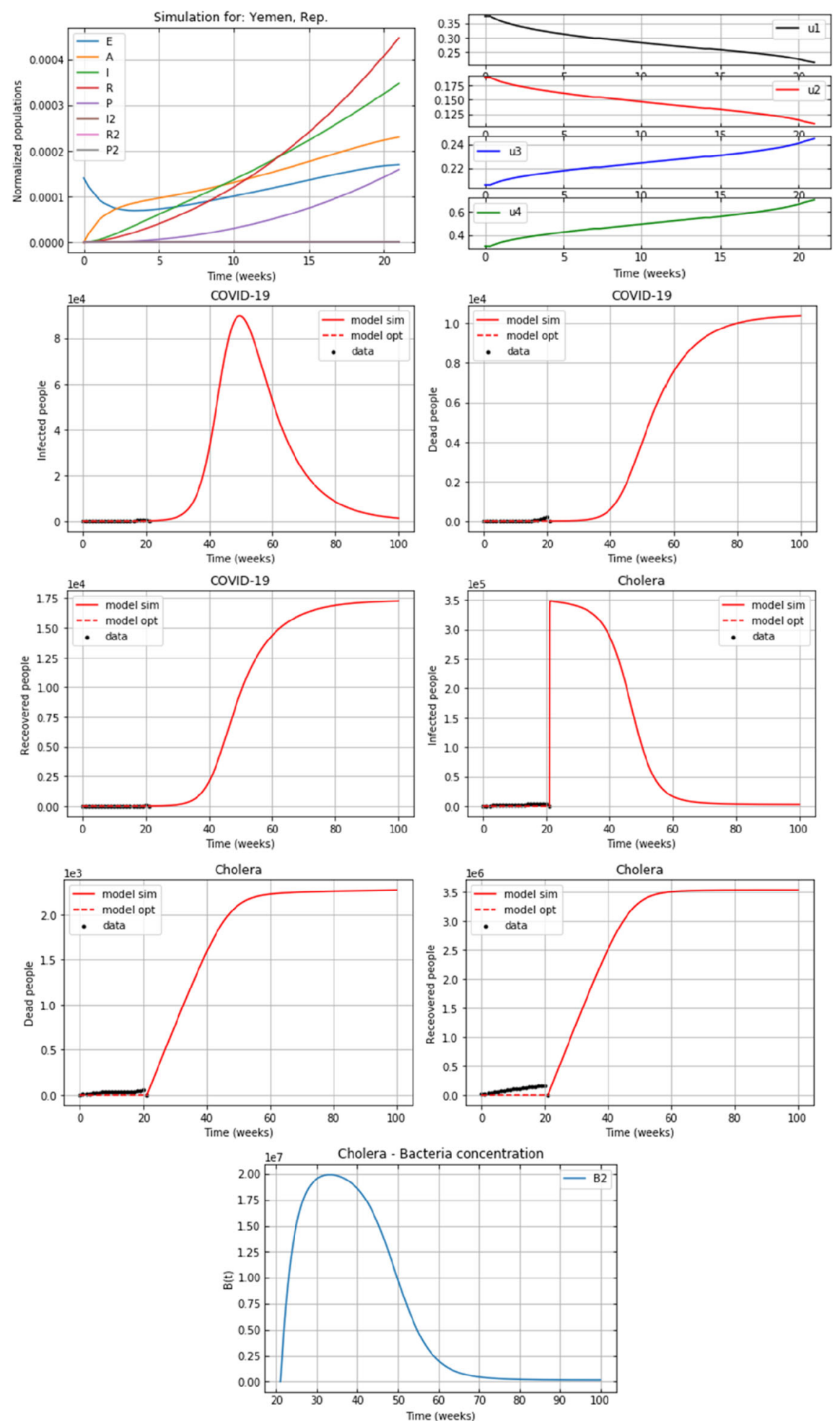

Figure 5 Results for policy 2 
Policy 3: (Max control)
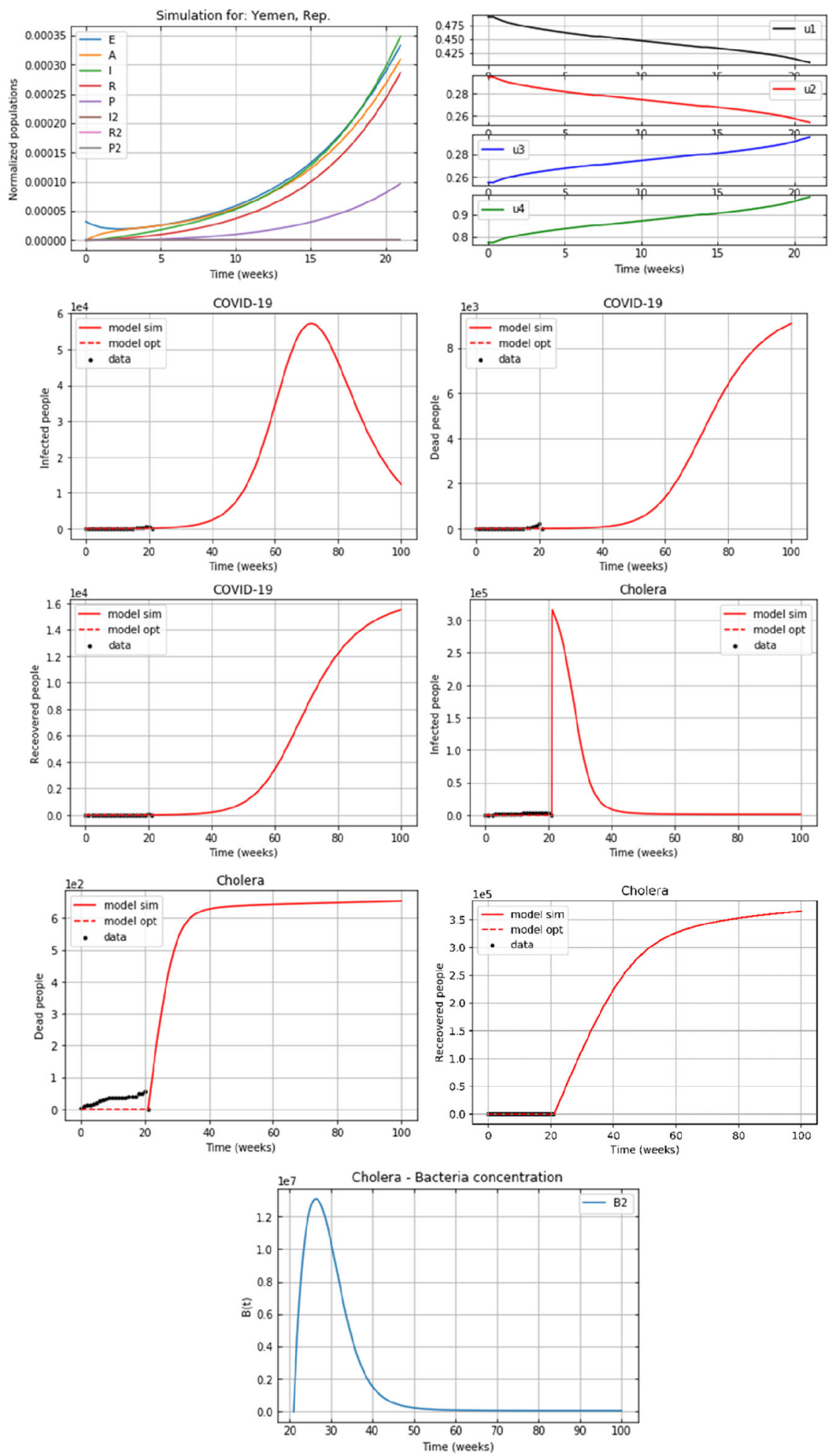

Figure 6 Results for policy 3 
Policy 4: (Mix control)
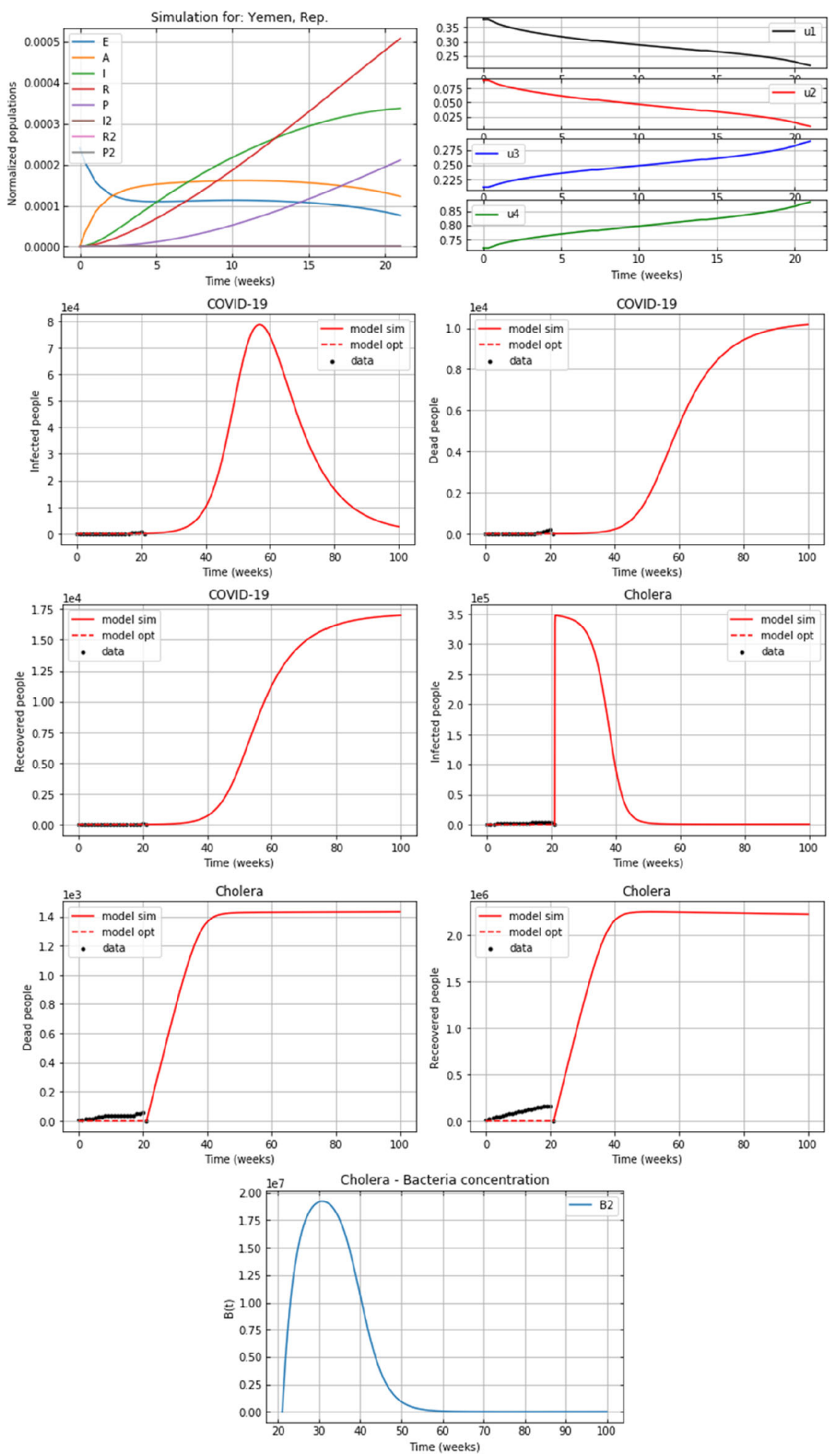

Figure 7 Results for policy 4 
Policy 5: Min peaks
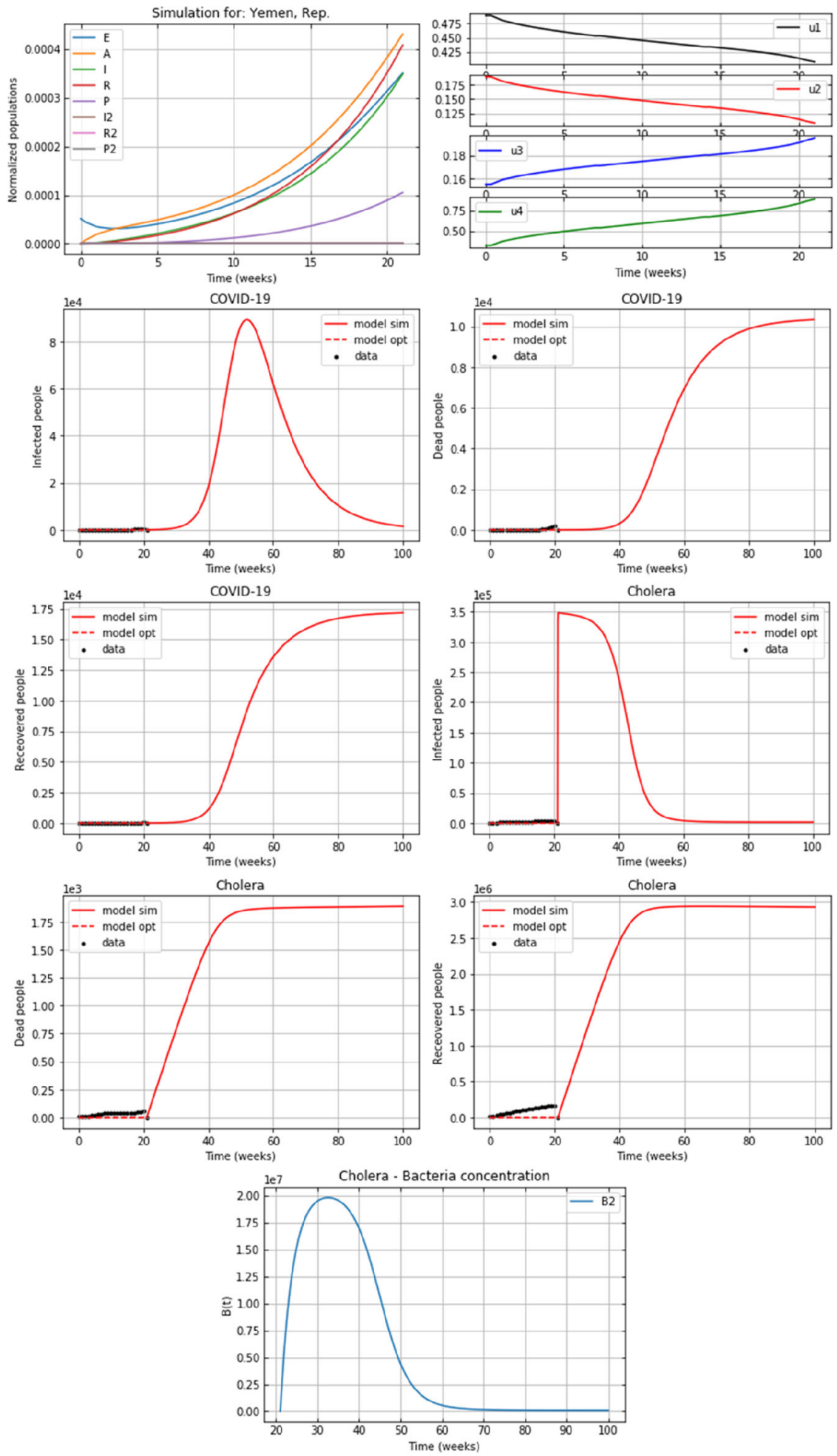

Figure 8 Results for policy 5 
Policy 6: Max peaks
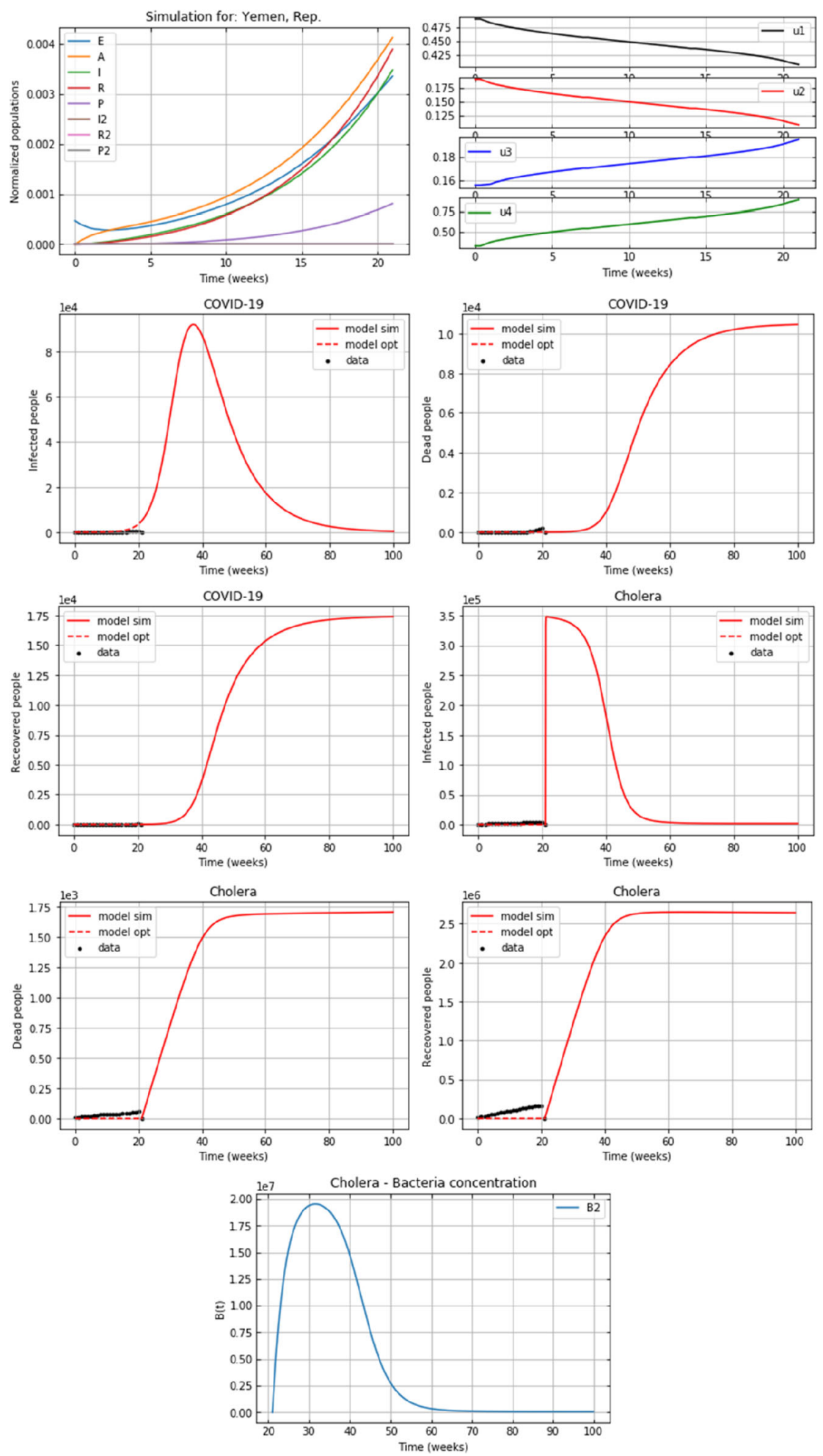

Figure 9 Results for policy 6 


\section{Appendix C}

Table 4 Ranks of the policies in various epidemiological classes

\begin{tabular}{|c|c|c|c|c|c|c|c|c|}
\hline & $1 /$ & $P_{1}$ & $R_{1}$ & $1 / 2$ & $P_{2}$ & $R_{2}$ & $B_{2}$ & $I_{1}+I_{2}$ \\
\hline 1 & Max control & Max control & Baseline & Max control & Max control & Baseline & Max control & Max control \\
\hline 2 & Mix control & Mix control & Max peaks & Mix control & Mix control & Med control & Mix control & Mix control \\
\hline 3 & Med control & Min peaks & Med control & Max peaks & Max peaks & Min peaks & Max peaks & Max peaks \\
\hline 4 & Min peaks & Med control & Min peaks & Min peaks & Min peaks & Max peaks & Min peaks & Min peaks \\
\hline 5 & Max peaks & Max peaks & Mix control & Med control & Med control & Mix control & Med control & Med control \\
\hline 6 & Baseline & Baseline & Max control & Baseline & Baseline & Max control & Baseline & Baseline \\
\hline
\end{tabular}

\section{Acknowledgements}

We would like to thank the editors of the journal as well as the anonymous reviewers for their valuable suggestions that make the paper stronger and more consistent.

\section{Funding}

The authors extend their appreciation to the Deanship of Scientific Research at King Saud University for funding this work through research group no. RG-1441-309.

\section{Availability of data and materials}

Not applicable.

\section{Competing interests}

The authors declare that they have no competing interests.

\section{Authors' contributions}

IMH, Conceptualization of this study, Data curation, Writing - Original draft preparation, Methodology, and Software. AF, Revision of draft preparation. AA, Revision of draft preparation. All authors read and approved the final manuscript.

\section{Authors' information}

Ibrahim M. Hezam received a PhD degree in operations research and decision support from Menoufia University, Egypt. He was a postdoctoral fellow in the Department of Industrial Engineering at Pusan National University, Busan, South Korea from January 2018 to January 2019. He is currently an assistant professor of operations research with the King Saud University, KSA. His research fields are artificial intelligence, optimization, network algorithms, operations research, and decision support. Abdelaziz Foul is an associate professor in the department of statistics and operations research, College of Science, King Saud University, Riyadh - Saudi Arabia. He obtained his PhD degree in operations research from Rensselaer Polytechnic Institute, Troy, New York. His research interests are linear and nonlinear optimization, facility location and allocation, optimal control of inventory systems. He has been the referee to many research journals. Adel Fahad Alrasheedi is an assistant professor in the Department of Statistics and Operations Research, College of Science, King Saud University, Riyadh, Saudi Arabia. He received a PhD degree in operations research from Edinburgh University, United Kingdom. His research interests are optimization, production/inventory systems, stochastic modelling, and optimal control.

\section{Publisher's Note}

Springer Nature remains neutral with regard to jurisdictional claims in published maps and institutional affiliations.

Received: 2 November 2020 Accepted: 1 February 2021 Published online: 15 February 2021

\section{References}

1. WHO EMRO: WHO EMRO | Outbreak update - cholera in Yemen, 11 October 2018. 38, 1207596 (2019)

2. Tian, J.P., Wang, J.: Global stability for cholera epidemic models. Math. Biosci. 232, 31-41 (2011). https://doi.org/10.1016/j.mbs.2011.04.001

3. Dangbé, E., Irépran, D., Perasso, A., Békollé, D.: Mathematical modelling and numerical simulations of the influence of hygiene and seasons on the spread of cholera. Math. Biosci. 296, 60-70 (2018). https://doi.org/10.1016/j.mbs.2017.12.004

4. Kobe, J., Pritchard, N., Short, Z., Erovenko, I.V., Rychtár̆, J., Rowell, J.T.: A game-theoretic model of cholera with optimal personal protection strategies. Bull. Math. Biol. (2018). https://doi.org/10.1007/s11538-018-0476-5

5. Opoku, N.K.-D.O., Afriyie, C.: The role of control measures and the environment in the transmission dynamics of cholera. Abstr. Appl. Anal. 2020, Article ID 2485979 (2020). https://doi.org/10.1155/2020/2485979

6. Berhe, H.W.: Optimal control strategies and cost-effectiveness analysis applied to real data of cholera outbreak in Ethiopia's Oromia region. Chaos Solitons Fractals 138, 1-14 (2020). https://doi.org/10.1016/j.chaos.2020.109933

7. Nishiura, H., Tsuzuki, S., Yuan, B., Yamaguchi, T., Asai, Y.: Transmission dynamics of cholera in Yemen, 2017: a real time forecasting. Theor. Biol. Med. Model. 14, 14 (2017). https://doi.org/10.1186/s12976-017-0061-x 
8. Yang, C., Wang, J: A cholera transmission model incorporating the impact of medical resources. Math. Biosci. Eng. 16, 5226-5246 (2019). https://doi.org/10.3934/mbe.2019261

9. Lemos-Paião, A.P., Silva, C.J., Torres, D.F.M.: A cholera mathematical model with vaccination and the biggest outbreak of world's history. AlMS Math. (2019). https://doi.org/10.3934/Math.2018.4.448

10. Lemos-Paião, A.P., Silva, C.J., Torres, D.F.M., Venturino, E.: Optimal control of aquatic diseases: a case study of Yemen's cholera outbreak. J. Optim. Theory Appl. 185, 1008-1030 (2020). https://doi.org/10.1007/s10957-020-01668-z

11. Carfora, M.F., Torcicollo, l.: Identification of epidemiological models: the case study of Yemen cholera outbreak. Appl. Anal. (2020). https://doi.org/10.1080/00036811.2020.1738402

12. Madubueze, C.E., Dachollom, S., Onwubuya, I.O.: Controlling the spread of COVID-19: optimal control analysis. Comput. Math. Methods Med. 2020, Article ID 6862516 (2020). https://doi.org/10.1155/2020/6862516

13. Khajii, B., Kada, D., Balatif, O., Rachik, M.: A multi-region discrete time mathematical modeling of the dynamics of Covid-19 virus propagation using optimal control. J. Appl. Math. Comput. 64, 255-281 (2020). https://doi.org/10.1007/s12190-020-01354-3

14. Perkins, T.A., España, G.: Optimal control of the COVID-19 pandemic with non-pharmaceutical interventions. Bull. Math. Biol. 82, 118 (2020). https://doi.org/10.1007/s11538-020-00795-y

15. Wickramaarachchi, W., Perera, S.S.N.: Optimal control measures to combat COVID 19 spread in Sri Lanka: a mathematical model considering the heterogeneity of cases (2020)

16. Yousefpour, A., Jahanshahi, H., Bekiros, S.: Optimal policies for control of the novel coronavirus disease (COVID-19) outbreak. Chaos Solitons Fractals 136, 109883 (2020). https://doi.org/10.1016/j.chaos.2020.109883

17. Lejarza, F., Stadtherr, M.A., Baldea, M., Tsay, C.: Modeling, state estimation, and optimal control for the US COVID-19 outbreak (2020)

18. Alkahtani, B.S.T., Jain, S.: Numerical analysis of COVID-19 model with constant fractional order and variable fractal dimension. Results Phys. 20, 103673 (2021). https://doi.org/10.1016/j.rinp.2020.103673

19. Khan, M.A., Atangana, A., Alzahrani, E., Fatmawati: The dynamics of COVID-19 with quarantined and isolation. Adv. Differ. Equ. 2020, 425 (2020). https://doi.org/10.1186/s13662-020-02882-9

20. Faraz, N., Khan, Y., Goufo, E.F.D., Anjum, A., Anjum, A.: Dynamic analysis of the mathematical model of COVID-19 with demographic effects. Z. Naturforsch. Teil C 75, 389-396 (2020). https://doi.org/10.1515/znc-2020-0121

21. Hezam, I.M., Nayeem, M.K., Foul, A., Alrasheedi, A.F.: COVID-19 vaccine: a neutrosophic MCDM approach for determining the priority groups. Results Phys. 20, 103654 (2021). https://doi.org/10.1016/j.rinp.2020.103654

22. Fatmawati, Khan, M.A., Bonyah, E., Hammouch, Z., Shaiful, E.M.: A mathematical model of tuberculosis (TB) transmission with children and adults groups: a fractional model. AlMS Math. 5, 2813-2842 (2020) https://doi.org/10.3934/math.2020181

23. Atangana, A., Jain, S.: A new numerical approximation of the fractal ordinary differential equation. Eur. Phys. J. Plus 133, 37 (2018). https://doi.org/10.1140/epjp/i2018-11895-1

24. Kabunga, S.K., Goufo, E.F.D., Tuong, V.H.: Analysis and simulation of a mathematical model of tuberculosis transmission in Democratic Republic of the Congo. Adv. Differ. Equ. 2020, 642 (2020). https://doi.org/10.1186/s13662-020-03091-0

25. Atangana, A., Goufo, E.F.D.: On the mathematical analysis of Ebola hemorrhagic fever: deathly infection disease in West African countries. BioMed Res. Int. 2014, Article ID 261383 (2014). https://doi.org/10.1155/2014/261383

26. Danane, J., Allali, K., Hammouch, Z: Mathematical analysis of a fractional differential model of HBV infection with antibody immune response. Chaos Solitons Fractals 136, 109787 (2020). https://doi.org/10.1016/j.chaos.2020.109787

27. Owusu, K.F., Goufo, E.F.D., Mugisha, S.: Modelling intracellular delay and therapy interruptions within Ghanaian HIV population. Adv. Differ. Equ. 2020, 401 (2020). https://doi.org/10.1186/s13662-020-02856-x

28. Jain, S., Atangana, A.: Analysis of Lassa hemorrhagic fever model with non-local and non-singular fractional derivatives. Int. J. Biomath. 11, 1850100 (2018). https://doi.org/10.1142/S1793524518501000

29. Agusto, F.B., Khan, M.A.: Optimal control strategies for denque transmission in Pakistan. Math. Biosci. 305, 102-121 (2018). https://doi.org/10.1016/j.mbs.2018.09.007

30. Li, J., Wang, L., Zhao, H., Ma, Z:: Dynamical behavior of an epidemic model with coinfection of two diseases. Rocky Mt. J. Math. 38, 1457-1479 (2008). https://doi.org/10.1216/RMJ-2008-38-5-1457

31. Gao, D., Porco, T.C., Ruan, S.: Coinfection dynamics of two diseases in a single host population. J. Math. Anal. Appl. 442, 171-188 (2016). https://doi.org/10.1016/j.jmaa.2016.04.039

32. Tang, B., Zhou, W., Xiao, Y., Wu, J.: Implication of sexual transmission of Zika on dengue and Zika outbreaks. Math. Biosci. Eng. 16, 5092-5113 (2019). https://doi.org/10.3934/mbe.2019256

33. Ghersheen, S., Kozlov, V., Tkachev, V.G., Wennergren, U.: Dynamical behaviour of SIR model with coinfection: the case of finite carrying capacity. Math. Methods Appl. Sci. 42, 5805-5826 (2019). https://doi.org/10.1002/mma.5671

34. Ghersheen, S., Kozlov, V., Tkachev, V., Wennergren, U.: Mathematical analysis of complex SIR model with coinfection and density dependence. Comput. Math. Methods 1(4), e1042 (2019). https://doi.org/10.1002/cmm4.1042

35. Khan, H., Gómez-Aguilar, J.F., Alkhazzan, A., Khan, A.: A fractional order HIV-TB coinfection model with nonsingular Mittag-Leffler law. Math. Methods Appl. Sci. 43, 3786-3806 (2020). https://doi.org/10.1002/mma.6155

36. Mushayabasa, S., Bhunu, C.P.: Is HIV infection associated with an increased risk for cholera? Insights from a mathematical model. Biosystems 109, 203-213 (2012). https://doi.org/10.1016/j.biosystems.2012.05.002

37. Okosun, K.O., Makinde, O.D.: A co-infection model of malaria and cholera diseases with optimal control. Math. Biosci. 258, 19-32 (2014). https://doi.org/10.1016/.j.mbs.2014.09.008

38. Okosun, K.O., Khan, M.A., Bonyah, E., Okosun, O.O.: Cholera-schistosomiasis coinfection dynamics. Optim. Control Appl. Methods 40, 703-727 (2019). https://doi.org/10.1002/oca.2507

39. Marimuthu, Y., Nagappa, B., Sharma, N., Basu, S., Chopra, K.K.: COVID-19 and tuberculosis: a mathematical model based forecasting in Delhi, India. Indian J. Tuberc. 67, 177-181 (2020). https://doi.org/10.1016/j.jitb.2020.05.006

40. Lam, L.T.M., Chua, Y.X., Tan, D.H.Y.: Roles and challenges of primary care physicians facing a dual outbreak of COVID-19 and dengue in Singapore. Fam. Pract. 37, 578-579 (2020). https://doi.org/10.1093/fampra/cmaa047

41. Goufo, E.F.D., Khan, Y., Chaudhry, Q.A.: HIV and shifting epicenters for COVID-19, an alert for some countries. Chaos Solitons Fractals 139, 110030 (2020). https://doi.org/10.1016/j.chaos.2020.110030

42. Hezam, I.M.: COVID-9 and unemployment: a novel bi-level optimal control model. Comput. Mater. Continua 67, 1153-1167 (2021). https://doi.org/10.32604/cmc.2021.014710 
43. Zhang, Z., Jain, S.: Mathematical model of Ebola and Covid-19 with fractional differential operators: non-Markovian process and class for virus pathogen in the environment. Chaos Solitons Fractals 140, 110175 (2020). https://doi.org/10.1016/j.chaos.2020.110175

44. Worldometers: Yemen population

Submit your manuscript to a SpringerOpen ${ }^{\circ}$ journal and benefit from:

- Convenient online submission

- Rigorous peer review

- Open access: articles freely available online

- High visibility within the field

- Retaining the copyright to your article

Submit your next manuscript at $\gg$ springeropen.com 